\title{
Precipitate-induced nonlinearities of diffusion along grain boundaries in Al-based alloys
}

\author{
Ankit Gupta, ${ }^{1, *}$ Vladislav Kulitcki,,${ }^{2,3}$ Bengü Tas Kavakbasi, ${ }^{2}$ Yulia Buranova, ${ }^{2}$ Jörg Neugebauer, ${ }^{1}$ \\ Gerhard Wilde, ${ }^{2}$ Tilmann Hickel, ${ }^{1}$ and Sergiy V. Divinski ${ }^{2, \dagger}$ \\ ${ }^{1}$ Max-Planck-Institut für Eisenforschung GmbH, D-40237 Düsseldorf, Germany \\ ${ }^{2}$ Institute of Materials Physics, University of Münster, D-48149 Münster, Germany \\ ${ }^{3}$ Laboratory of Mechanical Properties of Nanostructured Materials and Superalloys, Belgorod State University, 308015 Belgorod, Russia
}

(Received 24 January 2018; published 9 July 2018)

\begin{abstract}
The formation of precipitates in metallic alloys is determined by a two-way chemomechanical coupling. While the dependence on solute diffusion is apparent, the opposite effect, namely the impact of nanoprecipitates on the diffusion, is highlighted in the present paper. Using severe plastic deformation and post-deformation annealing of an Al-based alloy, different microstructures and sizes of $\mathrm{Al}_{3} \mathrm{Sc}$-based nanoprecipitates are produced. The enhanced diffusion along grain boundaries in the so-called C-type kinetic regime is used as a probe to ensure a well-defined distance of the diffusion path from the precipitates and to prohibit their evolution during measurements. Tracer measurements with the radioisotope ${ }^{57} \mathrm{Co}$ reveal a remarkable nonmonotonic dependence of the diffusion rates on the annealing temperature. It has been fully explained by an $a b$ initio informed phenomenological model that considers the elastic stress around the coherent and noncoherent precipitates.
\end{abstract}

DOI: 10.1103/PhysRevMaterials.2.073801

\section{INTRODUCTION}

Al-based alloys are attractive materials for various industrial applications owing to their low density combined with sufficient strength and ductility. Controlling their microstructure is a prerequisite for improving the mechanical properties. A strong microstructure refinement and formation of nanostructures by severe plastic deformation (SPD) is known as a promising technique [1-3]. For example, equal channel angular pressing (ECAP) at elevated temperatures has been applied to achieve superplastic properties of Al-based alloys [4].

The formation of nanoprecipitates in Al alloys can be achieved by dedicated annealing treatments at ambient pressure. One of the most prominent examples is the $L 1_{2}$ precipitate phase $\mathrm{Al}_{3} \mathrm{Sc}$. On one hand, the kinetics of the microstructure formation is determined by the diffusion rate of its solutes [5]. On the other hand, the formation of nanoprecipitates give rise to a coherency strain in the surrounding matrix, which modifies the diffusion rate of additional Sc atoms. The process of precipitate formation, as captured for example in temperature-time-transformation (ttt) diagrams, is therefore subject to a two-way chemomechanical coupling between the strain fields and the local chemistry (diffusion fluxes).

In the present paper, the relevance of the second coupling process is investigated, i.e., the effect of precipitates and the associated coherency/semicoherency strain fields onto the

\footnotetext{
*Corresponding author: gupta@mpie.de

${ }^{\dagger}$ Corresponding author: divin@wwu.de
}

Published by the American Physical Society under the terms of the Creative Commons Attribution 4.0 International license. Further distribution of this work must maintain attribution to the author(s) and the published article's title, journal citation, and DOI. solute diffusion. We therefore compare the results of an $a b$ initio informed phenomenological model for the strain dependent diffusion with dedicated tracer diffusion experiments. A complex Al-based alloy is chosen for the investigation. We chose $\mathrm{Mg}$ and $\mathrm{Sc}$ as main alloying components, since good experiences with nanoprecipitation have been made [6,7]. The ${ }^{57} \mathrm{Co}$ isotope is chosen as a convenient tracer element for this system. The volume diffusion coefficient of $\mathrm{Co}$ in pure $\mathrm{Al}$ at $320 \mathrm{~K}$ is about $10^{-30} \mathrm{~m}^{2} / \mathrm{s}$ as it follows from the Arrhenius parameters reported by Hood et al. [8]. Such a low value of Co diffusivity in bulk is due to the high activation energy of Co diffusion in $\mathrm{Al}$ (the experimentally reported range [8-12] and our $a b$ initio determined value are $1.68-1.82$ and $1.92 \mathrm{eV}$, respectively). Similarly low values can be assumed for $\mathrm{Co}$ volume diffusion in the $\mathrm{Al}$ alloys too, taking into account the relatively low amount of the alloying components and the typical values of the solute enhancement factors of Co [13].

SPD processed material and grain boundary diffusion are therefore chosen to have substantially higher diffusion coefficients at about room temperature. For example, an enhancement of grain boundary self-diffusion rates by orders of magnitude was established for ECAP-processed $\mathrm{Ni}$ [14] or Ti [15]. Using different annealing parameters, the fraction and the radii of the precipitates in the vicinity of the grain boundary can be systematically modified. The tracer diffusion method applied in the present study is highly sensitive to the state of the grain boundaries, grain boundary segregation, and grain boundary precipitation $[16,17]$. Thus, the determined diffusion coefficients represent a probe of a specific grain boundary state at a time scale at which the bulk diffusion of all substitutional elements in $\mathrm{Al}$ is frozen and the attained precipitate state is not modified during the measurements.

Material modification via SPD processing is not only limited to grain refinement but also includes modifications of the grain boundary (GB) kinetics, structure, and segregation 
TABLE I. The results of the diffusion experiments for Co GB diffusion in pure Al and the Al-Mg-Sc alloy at $320 \mathrm{~K}$ for three days, $D_{\mathrm{gb}}$. Before the diffusion experiments some samples were annealed at $T_{\text {an }}$ for the time $t_{\text {an }}$. The mean grain size $d$, dislocation density $\rho_{\mathrm{d}}$, and the average $\mathrm{Al}_{3}(\mathrm{Sc}, \mathrm{Zr})$-particle size $r_{\mathrm{av}}$ are specified. The parameter $\alpha^{*}$ is calculated using Eq. (12). The value of the GB diffusion coefficient measured for the ECAP-processed state (with $\alpha^{*}<1$ ) has to be corrected and the corrected value is given, $D_{\mathrm{gb}}^{\text {corr }}$ (see also text).

\begin{tabular}{|c|c|c|c|c|c|c|c|c|}
\hline Sample & $T_{\mathrm{an}}(\mathrm{K})$ & $t_{\mathrm{an}}(\mathrm{h})$ & $D_{\mathrm{gb}}\left(10^{-15} \mathrm{~m}^{2} / \mathrm{s}\right)$ & $d(\mu \mathrm{m})$ & $\rho_{\mathrm{d}}\left(10^{13} \mathrm{~m}^{-2}\right)$ & $r_{\mathrm{av}}(\mathrm{nm})$ & $\alpha^{*}$ & $D_{\mathrm{gb}}^{\text {corr }}\left(10^{-15} \mathrm{~m}^{2} / \mathrm{s}\right)$ \\
\hline pure Al & & & 17.5 & 500 & $10^{-2}-10^{-1}$ & & 6000 & \multirow{7}{*}{3.2} \\
\hline \multirow[t]{2}{*}{ as-cast } & & & 7.15 & 40 & 0.8 & $5-6$ & 7.5 & \\
\hline & & & 2.4 & 0.8 & 7 & 11 & 0.85 & \\
\hline ECAP- & 723 & 1 & 4.16 & 1.2 & $<1$ & $12-14$ & $>6$ & \\
\hline processed & 773 & 20 & 1.41 & 7 & $<1$ & 20 & $>6$ & \\
\hline \multirow[t]{2}{*}{ at $573 \mathrm{~K}$} & 823 & 50 & 3.88 & 10 & $<1$ & $30-40$ & $>6$ & \\
\hline & 823 & 200 & 9.0 & $>20$ & $<1$ & $>50$ & $>6$ & \\
\hline
\end{tabular}

$[18,19]$ simultaneously with the production of abundant point defects and dislocations in the processed material [20,21]. For example, the above mentioned enhanced grain boundary self-diffusion rates for ECAP-processed $\mathrm{Ni}$ [14] or Ti [15] were attributed to a deformation-modified (in pioneering works termed as "nonequilibrium" due to the heavy distortions induced locally and excess free volume [22-24]) state of the interfaces. Further, a strong segregation of $\mathrm{Mg}$ in our $\mathrm{Al}$ alloy is to be expected [25,26]. As a result, a sophisticated interplay of the hypothetical precipitation effect as well as deformation-induced grain boundary modifications and grain boundary segregation may be expected from the experimental point of view. In addition, to be able to compare with our $a b$ initio based model, we have undertaken a full analysis of the experimental data to disentangle different alternative contributions at the end of the paper.

\section{EXPERIMENTAL PROCEDURE}

\section{A. Material}

An Al-based alloy containing $4.6 \mathrm{wt} \% \mathrm{Mg}$ and $0.2 \mathrm{wt} . \%$ Sc (see Ref. [7] for additional alloying components as, e.g., $\mathrm{Mn}, \mathrm{Ti}, \mathrm{Zr}$ ) was produced by continuous casting, and ingots were homogenized by annealing at $(643 \pm 10) \mathrm{K}$ for $12 \mathrm{~h}$. The ingots were then extruded at $653 \mathrm{~K}$ imposing about $70 \%$ strain. The resulting rods were subjected to eight passes of ECAP processing at a temperature of $573 \mathrm{~K}$ with application of about $100 \mathrm{MPa}$ back pressure following the route $\mathrm{B}_{\mathrm{C}}$ [27]. The final grain size was about $500 \mathrm{~nm}$.

For a reference, coarse grained high-purity Al (99.999 wt.\%) was used. The polycrystalline Al sample material was subjected to $70 \%$ cold rolling, subsequently annealed at $873 \mathrm{~K}$ for $16 \mathrm{~h}$ and slowly cooled to room temperature to remove all effects of preparation procedure and assure nearly equilibrium conditions for GB segregation of residual impurities. The grain size was about $0.5 \mathrm{~mm}$.

\section{B. Sample preparation and characterization}

Discs of $10 \mathrm{~mm}$ diameter and $1.5 \mathrm{~mm}$ height were cut by spark erosion. Some samples were subjected to prediffusion heat treatments at selected temperatures for the times listed in Table I.

The microstructures were characterized by scanning electron microscopy (SEM) using a Nova Nano SEM 230 (FEI).
Orientation imaging microscopy was applied using electron backscatter diffraction (EBSD). For the EBSD analysis, the samples were mechanically ground, polished using diamond suspensions, finished with colloidal silica $(0.04 \mu \mathrm{m})$ until a mirrorlike surface finish was achieved, and finally electropolished using the $\mathrm{HClO}_{4}$ electrolyte.

Local microstructure analysis was performed with a transmission electron microscope (TEM) using a Libra $200 \mathrm{FE}$ TEM (Zeiss) and a JEOL JEM-2100 (both with an acceleration voltage of $200 \mathrm{kV}$ ). The disk-shaped samples for TEM analysis were cut from sections with the foil normal perpendicular to the extrusion direction in the central part of the processed materials. The samples from the ECAP-processed rods were cut perpendicularly to the extrusion direction. 1-mm-thick disks, $3 \mathrm{~mm}$ in diameter, were cut by spark erosion and mechanically polished down to the thickness of about $90 \mu \mathrm{m}$. The final thinning was done by chemical electropolishing (twin-jet electropolishing in a solution of $\mathrm{HNO}_{3}: \mathrm{CH}_{3} \mathrm{OH}$ with $1: 2$ ratio at $253 \mathrm{~K}$ ).

The sizes of the equiaxed grains were quantified by measuring the grain area and calculating an equivalent grain diameter by modeling each grain as a circle in accordance with the grain reconstruction method [28]. The size of the precipitates was estimated in bright-field TEM (BF-TEM) using at least ten arbitrarily selected micrographs, as it was employed in Ref. [29]. Additionally, dark field-TEM (DF-TEM) and high-angle annular dark-field scanning TEM (HAADF-STEM) micrographs were employed to measure the size of about 100 precipitates in each state. The total number of individual measurements for each condition was about 1000, see also Refs. [27,30]. The dislocation density was calculated in TEM by measuring the number of dislocations for the given intersection length and foil thickness.

The deformed microstructures were described in detail in previous publications $[4,7,31]$. Here we will present the most relevant details and the results for the microstructures relevant for subsequent diffusion measurements.

\section{Grain boundary tracer diffusion of ${ }^{57} \mathrm{Co}$}

Before the diffusion measurements, the surfaces of the samples were polished to a mirrorlike finish. Three to five microliters of the ${ }^{57} \mathrm{Co}$ tracer solution (272 days half-life and $122 \mathrm{keV} \gamma$-radiation) were dropped on the prepared surface and dried. The samples were placed in silica tubes and annealed. The annealing temperature was set to $320 \mathrm{~K}$ to be slightly 
above room temperature and the annealing time was fixed to three days. In order to eliminate the effects of surface and lateral diffusion, the sample diameter was reduced by about 1 $\mathrm{mm}$ by grinding after the diffusion annealing treatment.

The parallel serial sectioning was performed using Mylar foils (15 $\mu \mathrm{m}$ particle size) on a custom-built precision grinding machine. After removing a section, the sample was weighed by a microbalance. The thickness of each section was determined by the mass difference from the known density and radius of the sample. The relative specific radioactivity of each section (which is proportional to the tracer concentration) was determined by measuring the radioactive decays of the ${ }^{57} \mathrm{Co}$ tracer by a well-type intrinsic Ge $\gamma$-detector.

The grain boundary diffusion conditions were set to fulfill Harrison's C-type kinetic regime [32]. Therefore, the concentration profiles were plotted as the logarithm of the layer tracer concentration, $\ln \bar{c}$, vs the depth squared, $y^{2}$, and the corresponding diffusion coefficients, $D_{\mathrm{gb}}$, were determined using the standard Gaussian solution [33],

$$
D_{\mathrm{gb}}=\frac{1}{4 t}\left(-\frac{\partial \ln \bar{c}}{\partial y^{2}}\right)^{-1},
$$

where $t$ is the diffusion time. The slope of the above plot allows a direct determination of $D_{\mathrm{gb}}$.

\section{DENSITY FUNCTIONAL THEORY (DFT) METHODOLOGY}

In order to study the chemomechanical coupling during precipitation formation, the DFT computations address the impact of coherency strain on the bulk diffusion. We would like to use the experimentally observed relationship between the coherency strain around the particles and the diffusivities along the general high-angle GBs as a benchmark. For the comparison, we use the experimentally established correlation between the activation energies of GB diffusion, $Q_{\mathrm{gb}}$, (measured directly in the corresponding C-type regime for solute GB diffusion) and those for bulk diffusion in the same matrix, $Q_{\mathrm{v}}$, which is in metals, $Q_{\mathrm{gb}} \approx(0.4-0.6) Q_{\mathrm{v}}$ [33,34]. Moreover, pressure-dependent measurements of $\mathrm{Zn} \mathrm{GB}$ diffusion in $\mathrm{Al}$ yield similar values of the activation volume as expected for bulk diffusion in $\mathrm{Al}$, about $0.8 \Omega$ and $0.9 \Omega$, respectively [35] ( $\Omega$ is the atomic volume).

The total energy calculations were performed employing the projector augmented wave (PAW) method as implemented in the Vienna ab initio simulation package (VASP) [36,37] with energetics based on density functional theory (DFT). The generalized gradient approximation (GGA) as parameterized by Perdew, Burke, and Ernzerhof (PBE) [38] was used to describe the electronic exchange and correlation effects. The integration over the Brillouin zone was performed using the MonkhorstPack [39] scheme with $12 \times 12 \times 12$ (for 32 atoms) and $8 \times$ $8 \times 8$ (for 108 atoms) reciprocal-space $k$ mesh centered around the $\Gamma$ point and utilizing the Methfessel-Paxton scheme [40] with a thermal smearing width of $0.15 \mathrm{eV}$. The plane-wave energy cutoff was set to $400 \mathrm{eV}$. An energy of $10^{-6} \mathrm{eV}$ was used as a convergence criterion for the self-consistent electronic loop.

The minimum energy path and the diffusion barrier for a vacancy-mediated nearest neighbor jump of Co in a face centered cubic (fcc) Al matrix is obtained employing the climb- ing image nudged elastic band (CI-NEB) method [41,42] as implemented in the VTST code [43]. We have used $2 \times 2 \times 2$ and $3 \times 3 \times 3 \mathrm{fcc}$ supercells (32 and 108 atoms, respectively). A tolerance value of $0.05 \mathrm{eV} / \AA$ was chosen for the force components along and perpendicular to the tangent to the reaction path. A total of five images/configurations (in addition to the initial and the final state) were considered to generate the minimum energy path in the configuration space. As expected, the saddle point lies midway along this minimum energy path along the [110] direction [see Fig. 6(b)].

In order to address the experimentally-relevant effect of hydrostatic pressure, the diffusion barrier is also calculated in a hydrostatically strained Al matrix for different values of the lattice parameter. The strain dependence of the diffusion barrier was obtained by performing total energy calculations for 13 equally spaced hydrostatic strain $(\epsilon)$ values ranging between $\pm 1.5 \%(-1.5,-1.25,-1, \ldots, 1,1.25,1.5)$ where $\epsilon=\left(a-a_{\mathrm{eq}}\right) / a_{\mathrm{eq}} . a_{\mathrm{eq}}$ is the computed equilibrium lattice parameter of pure $\mathrm{Al}(4.04 \AA)$ as obtained within GGA. With this definition of $\epsilon$, negative and positive values correspond to compressive and dilatational strains, respectively. The internal atomic coordinates were fully relaxed during the total energy calculations maintaining the strained volume.

A $2 \times 2 \times 8$ supercell was used to set up a coherent planar (100) oriented interface between $\mathrm{Al}$ and $\mathrm{Al}_{3} \mathrm{Sc}$ with equal volume fractions of both phases. The interfacial in-plane lattice constant was fixed while relaxing out of plane lattice vectors. The ionic positions were relaxed in all directions until the forces on the ions were below $10^{-5} \mathrm{eV} / \AA$. The above procedure was repeated for in-plane lattice constant values varying from 4.00-4.14 A.

The Co segregation energy at the $\mathrm{Al} / \mathrm{Al}_{3} \mathrm{Sc}$ interface is defined as

$$
E_{\mathrm{seg}}=\left\{E_{\mathrm{Co}}^{\mathrm{Al} / \mathrm{Al}_{3} \mathrm{Sc}}+E^{\mathrm{Al}, \text { bulk }}\right\}-\left\{E^{\mathrm{Al} / \mathrm{Al}_{3} \mathrm{Sc}}+E_{\mathrm{Co}}^{\mathrm{Al}, \text { bulk }}\right\},
$$

where $E_{\mathrm{Co}}^{\mathrm{Al} / \mathrm{Al}_{3} \mathrm{Sc}}$ and $E^{\mathrm{Al} / \mathrm{Al}_{3} \mathrm{Sc}}$ are the total energies of supercells containing a coherent planar interface between $\mathrm{Al}$ and $\mathrm{Al}_{3} \mathrm{Sc}$ with and without a $\mathrm{Co}$ atom replacing an $\mathrm{Al}$ atom at the interface; $E_{\mathrm{Co}}^{\mathrm{Al} \text {,bulk }}$ and $E^{\mathrm{Al} \text {,bulk }}$ are the total energies of bulk $3 \times 3 \times 3$ fcc Al supercells with and without a Co atom in a strain-free state. In Eq. (2), a negative value of $E_{\text {seg }}$ represents favorable segregation and vice versa. We performed convergence tests for the Co segregation energy as a function of distance from the interface with respect to the supercell size varying from $2 \times 2 \times 4$ to $2 \times 2 \times 10$ for a fixed inplane strain condition. For the employed supercell $(2 \times 2 \times 8)$, the segregation energies were found to be converged below $30 \mathrm{meV}$.

\section{EXPERIMENTAL RESULTS}

The GB diffusion-related branches of the penetration profiles of ${ }^{57} \mathrm{Co}$ tracer diffusion in the $\mathrm{Al}$ alloy for the as-cast and different processed states shown in Fig. 1 are found to follow a linear behavior in the given coordinates. The first near-surface points are affected by the grinding procedure and omitted from the subsequent analysis.

The exact values of the volume diffusion coefficients are not relevant, since at low temperatures the effective GB width, 


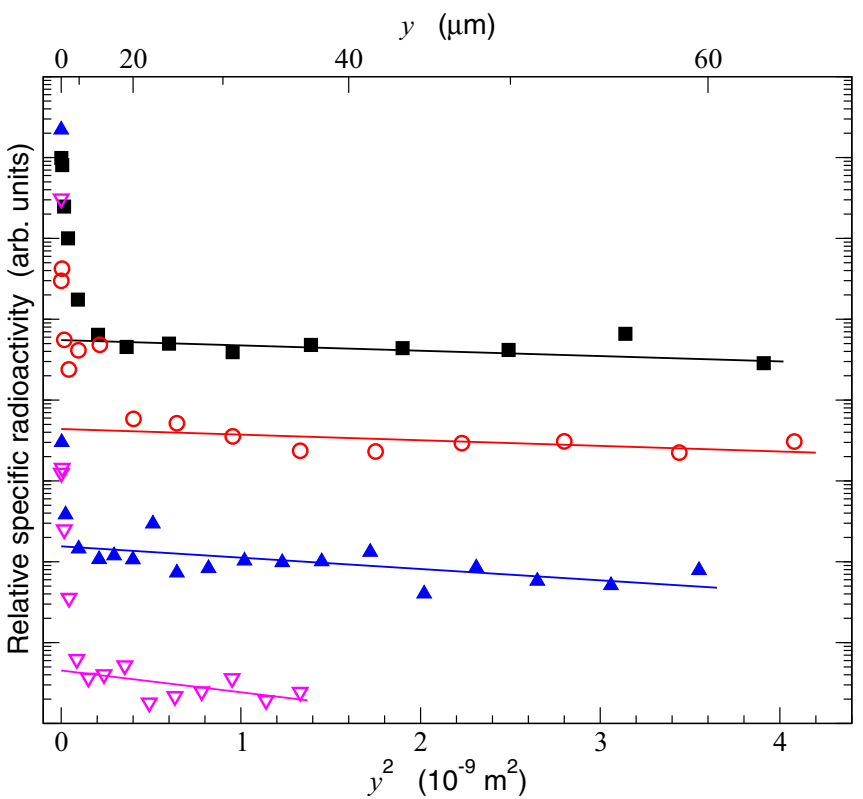

FIG. 1. The penetration profiles measured for ${ }^{57} \mathrm{Co}$ tracer diffusion at room temperature in the $\mathrm{Al}$ alloy in the following states: as-cast (squares), ECAP-processed (circles), ECAP-processed and annealed at $623 \mathrm{~K}$ for $1 \mathrm{~h}$ (triangles up) and at $773 \mathrm{~K}$ for $20 \mathrm{~h}$ (triangles down). $y$ is the penetration depth. The penetration profiles are shifted along the ordinate axis for a convenient presentation.

$s \cdot \delta$, is significantly larger than the effective diffusion length in the bulk $\sqrt{D_{\mathrm{v}} t}$ (the condition for C-type diffusion [33]), i.e., the Le Claire parameter

$$
\alpha=\frac{s \cdot \delta}{2 \sqrt{D_{\mathrm{v}} t}}
$$

is significantly larger than unity. In Eq. (3), $D_{\mathrm{v}}$ is the volume diffusion coefficient of $\mathrm{Co}$ in $\mathrm{Al}, t$ is the diffusion time, $s$ is the segregation factor defined as the ratio of the Co concentration in the GB and in the bulk close to the GB, and $\delta$ is the GB diffusional width. The measured value of the width $\delta$ for fcc metals is about $0.5 \mathrm{~nm}[44,45]$ and the segregation factor for $\mathrm{Co}$ at $\mathrm{Al}$ grain boundaries can be expected to be $\geqslant 1$. Note that simple estimates prove that $\alpha>100$ already for $s^{\mathrm{Co}}=1$.

The measured diffusion coefficients, $D_{\mathrm{gb}}$, of Co diffusion in the present Al-based alloy in different investigated states are listed in Table I. It should be noted that the experimental conditions for Co GB diffusion in the ECAP-processed state do not correspond to the true C-type kinetics due to dislocationenhanced diffusion and a correction is necessary, see Table I and Sec. VI B 2, Model 2 below. We observe that after ECAP processing, the Co GB diffusion rate drops with respect to the value in the as-cast state by a factor of two to three. To be more specific, the measured diffusion coefficients $D_{\mathrm{gb}}$ for Co in the ECAP-processed Al alloy are plotted in Fig. 2(a) as a function of the post-deformation annealing temperature $T_{\text {an }}$ (symbols) and compared with the value measured in the as-deformed alloy (dot-dashed line).

There is a remarkable trend with a strongly nonmonotonous dependence of the Co GB diffusion coefficients in the ECAPprocessed ultrafine grained $\mathrm{Al}$ alloy on the annealing parameters, in fact they first decrease and then increase again reaching almost the level which is characteristic for the Co GB diffusion rates in as-cast or ECAP-processed states.

In order to provide a reliable reference, Co GB diffusion in well-annealed coarse-grained pure $\mathrm{Al}$ was measured too (Table I). Here, the Co GB diffusion is substantially faster than in all considered states of the Al-based alloy. This feature can be attributed to $\mathrm{Mg}$ segregation, as discussed later.

\section{DISCUSSION}

\section{A. Correlation of experimental diffusion trends and precipitate formation}

It is obvious that the nonmonotonous dependence shown in Fig. 2(a) cannot be attributed to a usual relaxationlike behavior, since annealing at $823 \mathrm{~K}$ for long times increases

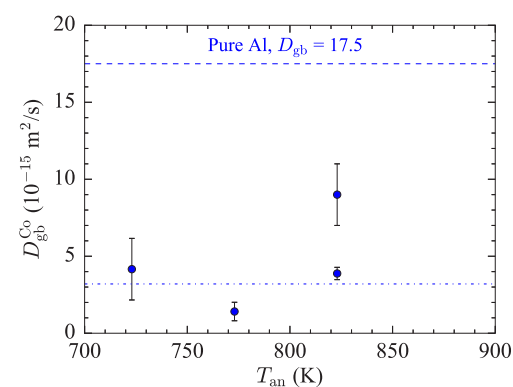

(a)

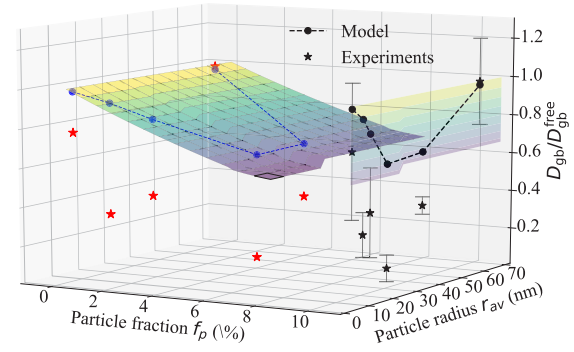

(b)

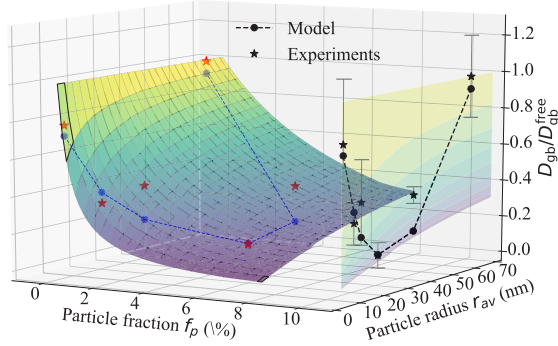

(c)

FIG. 2. (a) The diffusion coefficient of Co in the ECAP-processed Al alloy as a function of the post-deformation annealing temperature for varying annealing times (see Table I). The GB diffusion coefficient measured for Co in the ECAP-deformed alloy (without annealing) is shown by the dot-dashed line ( $D_{\mathrm{gb}}^{\text {corr }}$ in Table I) and that in pure $\mathrm{Al}$ is represented by the dashed line for comparison purposes. (b) and (c) $3 \mathrm{D}$ surface showing the predicted variation of the diffusion coefficient of Co (normalized with respect to the diffusion coefficient in the stress-free state, $D_{\mathrm{gb}} / D_{\mathrm{gb}}^{\mathrm{free}}$ ) with the particle size and the particle fraction for Co segregation factor $k_{s}=1$ and 1000, respectively (see Appendix A). The filled star symbols represent the measured values (from Table I with $D_{\mathrm{gb}}^{\text {free }}=9 \times 10^{-15} \mathrm{~m}^{2} / \mathrm{s}$ ). The dependence of the diffusion coefficient on the particle size includes an implicit dependence on the associated elastic fields produced by the particles as described by the model outlined in Sec. VE and Appendix A. The dashed blue line connects the model predictions on the 3D surface for the experimentally determined values of $f_{p}$ and $r_{\mathrm{av}}$. The measured and predicted values (for known $f_{p}$ and $r_{\mathrm{av}}$ values) are also projected onto the front plane with the black dashed line joining the model predictions. 


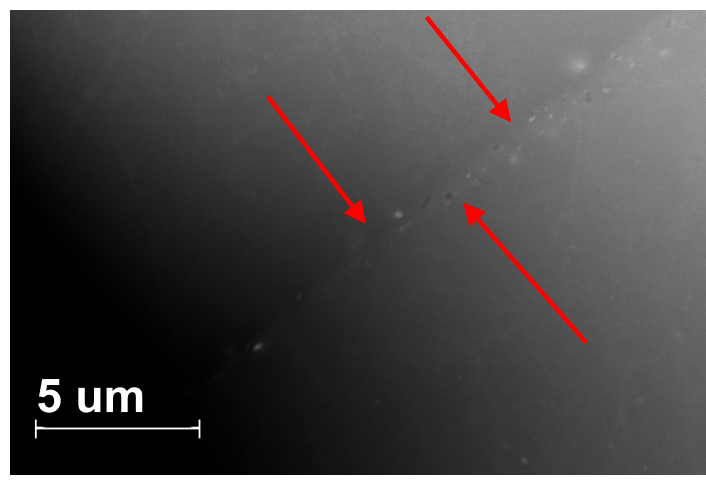

(a)

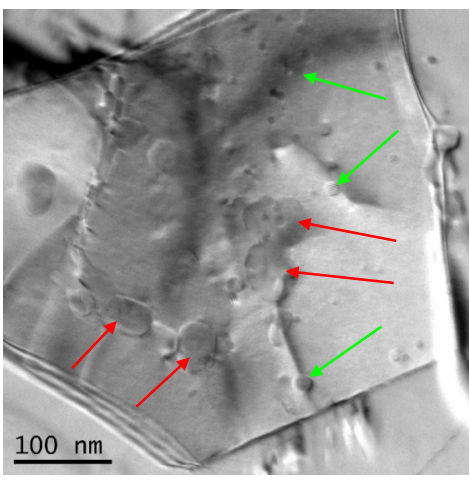

(b)

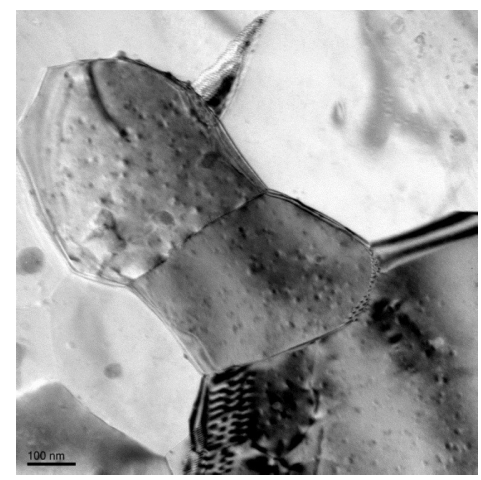

(c)

FIG. 3. (a) HAADF-STEM image of a GB in the $\mathrm{Al}$ alloy in the as-cast state. The high concentration of $\mathrm{Al}_{6} \mathrm{Mn}$ precipitates on the GB is indicated by red arrows. (b) TEM image of a single grain in the alloy after ECAP processing at $573 \mathrm{~K}$. $\mathrm{Al}_{6} \mathrm{Mn}$ and $\mathrm{Al}_{3} \mathrm{Sc}$ precipitates are indicated by red and green arrows, respectively. (c) Distribution of $\mathrm{Al}_{3} \mathrm{Sc}$-based precipitates in grains and around interfaces in the $\mathrm{Al}$-based alloy after ECAP at $573 \mathrm{~K}$ and annealing at $723 \mathrm{~K}$ for 1 hour.

the diffusion coefficient, although annealing treatments at lower temperatures decrease it. Instead, according to our consideration in Sec. I, we will explain this feature in the following by the formation of particles. In our previous work on $\mathrm{Ni}$ and $\mathrm{Ti}$ GB diffusion in NiTi alloy, a retardation of the GB diffusion rate was reported at high temperatures and was related to the precipitation of $\mathrm{Ni}_{4} \mathrm{Ti}_{3}$ particles at the interfaces [46]. In the present work, we analyze the effect of $\mathrm{Al}_{3} \mathrm{Sc}$ precipitates on GB diffusion in the Al-based severely deformed alloy quantitatively.

Large precipitates with an average size of about $200 \mathrm{~nm}$ are found at interfaces, as substantiated by the HAADFSTEM image, Fig. 3(a). The bright-appearing particles in these conditions are characterized by larger $Z$ numbers of the scattering atoms than the matrix (Al) and correspond to the intermetallic $\mathrm{Al}_{6} \mathrm{Mn}$ precipitates possessing an orthorhombic crystal structure [47]. In the as-cast state, these precipitates are mainly concentrated near GBs, with a relatively large (>300 nm) average distance between them. Since they are in addition incoherent with the matrix, the impact of these particles on the measured Co GB diffusion is expected to be low.

Figures 3(b) and 3(c) show a typical contrast (including the appearance of Moiré patterns) at GBs in different states. One clearly sees that the interfaces are straight and flat and appear as relaxed interfaces, differently from the GB appearance in ECAP-processed Ni [14] or Ti [15], for example. This finding agrees perfectly with the results of our GB diffusion measurements, which substantiate an absence of deformation-enhanced GB diffusion in the Al-based alloy under consideration unlike the case for $\mathrm{Ni}$ or $\mathrm{Ti}$ (Sec. VIB).

After ECAP processing, $\mathrm{Al}_{6} \mathrm{Mn}$ and $\mathrm{Al}_{3} \mathrm{Sc}$ precipitates are more uniformly distributed in the grain interiors [Fig. 3(b)]. The $\mathrm{Al}_{6} \mathrm{Mn}$ particles are of a size of about $70 \mathrm{~nm}$ and are predominantly located at dislocations and sub-boundaries. The $\mathrm{Al}_{3} \mathrm{Sc}$-based particle density near the GBs is estimated to be about $10^{2} \mu \mathrm{m}^{-3}$, which is by two orders of magnitude lower than the total density (about $10^{4} \mu \mathrm{m}^{-3}$ ). Thus the particles are mainly distributed in the grain interiors, keeping the GBs relatively free.

As a result of post-deformation annealing, the particles grow and the $\mathrm{Al}_{3} \mathrm{Sc}$ precipitates approach an average size of
$40 \mathrm{~nm}$ and more (Table I). Whereas $\mathrm{Al}_{3} \mathrm{Sc}$ precipitates are fully coherent in the ECAP-processed state (their average size is about $11 \mathrm{~nm}$ ), they become semicoherent after annealing treatment as a result of growth and finally loose their coherency after annealing at high temperatures.

The critical size for coherency loss is determined from elasticity theory $[48,49]$. It is obtained by equating the decrease in the elastic strain energy of the precipitate-matrix system due to the coherency loss and the increase in the interfacial energy due to the formation of a misfit dislocation at the interface (see Appendix B for details). The strain energy due to the mismatch $\epsilon=\left(a_{\mathrm{p}}-a_{\mathrm{m}}\right) / a_{\mathrm{m}}$ between the lattice parameters of the matrix and the precipitate, respectively, grows with the precipitate size and beyond a critical value results in a coherency loss. At ambient conditions, the equilibrium lattice parameter of $\mathrm{Al}_{3} \mathrm{Sc}(\approx 4.124 \AA)$ is slightly larger than that of Al $(\approx 4.072 \AA)$. However, considering the contribution of all finite-temperature excitations (including full anharmonicity), our calculated coefficient of thermal expansion of Al increases more rapidly with temperature as compared to that of $\mathrm{Al}_{3} \mathrm{Sc}$, which effectively decreases the lattice mismatch as temperature increases. The resulting misfit at the relevant annealing temperatures $773-823 \mathrm{~K}$ yields a calculated critical radius between 23-26 nm for $\mathrm{Al}_{3} \mathrm{Sc}$ [using Eq. (B3)], agreeing well with our experimentally observed range of $20-30 \mathrm{~nm}$ and the previously reported values [48,50-55]. The critical particle size

TABLE II. The summary of critical sizes for $\mathrm{Al}_{3} \mathrm{Sc}$-based precipitates in the case of pure $\mathrm{Al}$ and the $\mathrm{Al}-\mathrm{Mg}$ alloy. The calculations were performed using the data from Ref. [57].

\begin{tabular}{lccc}
\hline \hline Matrix & Particle & Mismatch $(\%)$ & $\begin{array}{c}\text { Critical particle } \\
\text { size }(\mathrm{nm})\end{array}$ \\
\hline \multirow{4}{*}{$\mathrm{Al}$} & $\mathrm{Al}_{3} \mathrm{Sc}$ & 1.33 & 15.2 \\
& $\mathrm{Al}_{3}(\mathrm{Sc}, \mathrm{Zr})$ & 1.14 & 17.7 \\
& $\mathrm{Al}_{3}(\mathrm{Sc}, \mathrm{Ti})$ & 0.64 & 31.6 \\
$\mathrm{Al}+4.48 \% \mathrm{Mg}$ & $\mathrm{Al}_{3} \mathrm{Sc}$ & 0.87 & 23.4 \\
& $\mathrm{Al}_{3}(\mathrm{Sc}, \mathrm{Zr})$ & 0.67 & 30.4 \\
& $\mathrm{Al}_{3}(\mathrm{Sc}, \mathrm{Ti})$ & 0.18 & 113.0 \\
\hline
\end{tabular}



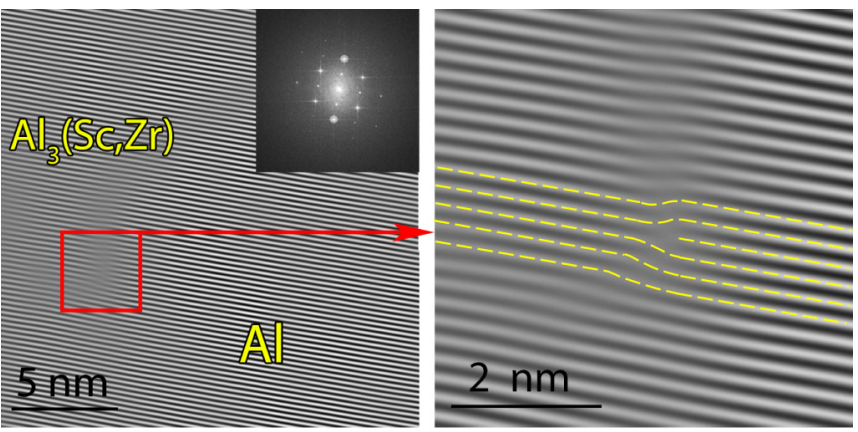

FIG. 4. FFT analysis of the $\mathrm{Al} / \mathrm{Al}_{3}(\mathrm{Sc}, \mathrm{Zr})$ interface in the (002)$\mathrm{Al}$ Bragg maxima in the $\mathrm{Al}$ alloy after ECAP processing at $573 \mathrm{~K}$ and subsequent annealing at $673 \mathrm{~K}$ for $1 \mathrm{~h}$. A misfit dislocation is visualized by the yellow lines.

is found to increase with temperature, a trend consistent with the $\mathrm{Al} / \mathrm{Al}_{3} \mathrm{Sc}$ lattice misfit which decreases with temperature.

A more detailed chemical analysis should take into account that the Al alloy under investigation contains up to 4.5 wt. $\%$ of $\mathrm{Mg}$, which increases the lattice parameter of the matrix [56] thereby decreasing the misfit. Moreover, in the ECAP-processed state the $\mathrm{Al}_{3} \mathrm{Sc}$ particles contain $\mathrm{Zr}$ (and sometimes Ti) [7] that decreases the lattice parameter of the precipitates and hence the misfit. We performed an analysis using the data from Ref. [57] (see Table II). The results show that the $\mathrm{Al}_{3}(\mathrm{Sc}, \mathrm{Zr})$ precipitates in the $\mathrm{Al}-4.5 \mathrm{wt} . \% \mathrm{Mg}$ matrix start to loose their coherency with the matrix at the sizes of about $20-30 \mathrm{~nm}$ (depending on the $\mathrm{Zr}$ content). The Ticontaining $\mathrm{Al}_{3}(\mathrm{Sc}, \mathrm{Ti})$ precipitates will not be analyzed further, since their volume fraction is found to be low [7].

Figure 4 shows a dislocation analysis of the interface structure for an $\mathrm{Al}_{3}(\mathrm{Sc}, \mathrm{Zr})$ precipitate. The size of the particle is about $22 \mathrm{~nm}$ and the appearance of misfit dislocations has to be expected according to the analysis (Table II). The precipitate is not perfectly spherical, which correlates with a relatively low content of $\mathrm{Zr}$ atoms [7]. A fast Fourier transformation (FFT) image made in the (002)-Al Bragg maxima indeed verifies the existence of a misfit dislocation between the matrix and the particles (red arrow in Fig. 4).

All these observations are qualitatively consistent with measured diffusion coefficients $D_{\mathrm{gb}}$, which have been plotted as a function of radius of the $\mathrm{Al}_{3} \mathrm{Sc}$-based precipitates, $R_{\mathrm{av}}$, in Fig. 2(c). A strongly nonmonotonous dependence is seen where the Co GB diffusion coefficient first decreases with increasing radius, reaching a critical value at about $R_{\mathrm{av}} \approx$ 20-30 nm, followed by an increase and approaching the value of $D_{\mathrm{gb}}$ for small precipitates. Note that this range exactly corresponds to the critical size of $\mathrm{Al}_{3} \mathrm{Sc}$ and $\mathrm{Al}_{3}(\mathrm{Sc}, \mathrm{Zr})$ precipitates when the appearance of misfit dislocations and a gradual loss of the precipitate/matrix coherency are expected (Table II). In order to a make a quantitative analysis, we analyze in the following the effect of these precipitates on GB diffusion by an $a b$ initio informed phenomenological model.

\section{B. Maxwell-Garnett model}

The direct influence of the particles on the net diffusion flux along GBs in the $\mathrm{Al}$ alloy is described within the frame-

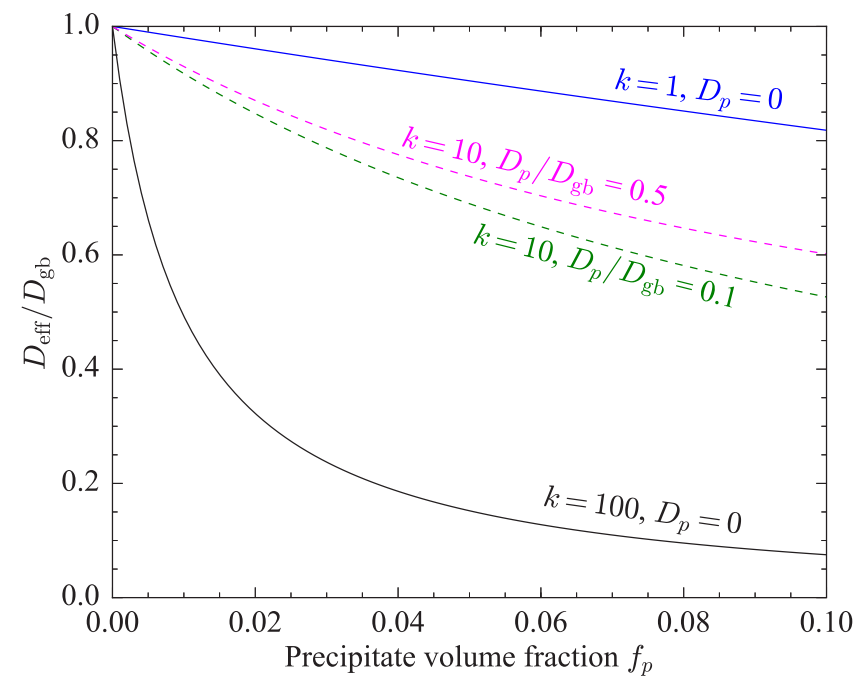

FIG. 5. Diffusion retardation, $D_{\text {eff }} / D_{\mathrm{gb}}$, in precipitated GBs as a function of the precipitate volume fraction. The dependencies were calculated according to Eq. (4) for different values of the enrichment factor $k$ and keeping $D_{\mathrm{p}}=0$ (solid lines) and for the case of $k=10$ and allowing diffusion inside of particles, $D_{\mathrm{p}} \neq 0$ (dashed lines).

work of the modified Maxwell-Garnett approach [58,59] (see Appendix A),

$$
\frac{D^{\mathrm{eff}}}{D_{\mathrm{gb}}}=\frac{1}{1+f_{\mathrm{p}}(k-1)} \times \frac{\left(1-f_{\mathrm{p}}\right)+k\left(1+f_{\mathrm{p}}\right) \frac{D_{\mathrm{p}}}{D_{\mathrm{gb}}}}{\left(1+f_{\mathrm{p}}\right)+k\left(1-f_{\mathrm{p}}\right) \frac{D_{\mathrm{p}}}{D_{\mathrm{gb}}}} .
$$

Here, $f_{\mathrm{p}}$ is the volume fraction of precipitates. The precipitates are characterized by their diffusion coefficient $D_{\mathrm{p}} . k\left[=\frac{c}{c_{m}}\right.$, see Appendix A and Fig. 9(b)] is the enrichment factor quantifying the favorable occupation of tracer Co atoms of the sites near the $\mathrm{Al} / \mathrm{Al}_{3} \mathrm{Sc}$ or $\mathrm{Al} / \mathrm{Al}_{3}(\mathrm{Sc}, \mathrm{Zr})$ interfaces. The interfaces in fact represent triple lines as intersections between the grain boundary plane and particle surface within Al grain boundaries.

The sole effect of the precipitate volume fraction (for a fixed set of $k$ and $D_{p}$ neglecting for a moment the stress fields completely) on the effective diffusion coefficient is illustrated in Fig. 5. Figure 5 shows that even in the case of a low (about $1 \%$ ) fraction of impermeable precipitates $\left(D_{\mathrm{p}}=0\right)$, a strong segregation of tracer atoms to the $\mathrm{GB} /$ precipitate triple lines $(k=100)$ would slow down the GB diffusion rate by a factor of two. This finding already agrees with the experimental observations in the present measurements [Fig. 2(c)]. Note that for the critical size of the $\mathrm{Al}_{3} \mathrm{Sc}$ precipitates of about $20 \mathrm{~nm}$, Table I, and the average distance between them about $50 \mathrm{~nm}$ (the measured precipitate density of $10^{2} \mu \mathrm{m}^{-1}$ in the near GB region), the particle fraction is estimated at even $6 \%$ in the case of 2D GB diffusion.

Since the melting point of the $\mathrm{Al}_{3} \mathrm{Sc}$ phase [6] is about $1320^{\circ} \mathrm{C}$, one can safely exclude any diffusion penetration of $\mathrm{Co}$ atoms inside the precipitates in the present experiments at near room temperature and use $D_{\mathrm{p}}=0$ inside of particles. This is a valid assumption also due to the high energy barriers associated with the Co diffusion inside the $\mathrm{Al}_{3} \mathrm{Sc}$ particles. The migration barriers of Co for vacancy mediated jumps towards and away 
from the first nearest-neighbor Sc site in $L 1_{2}$ ordered $\mathrm{Al}_{3} \mathrm{Sc}$ computed using CI-NEB simulations are 0.49 and $1.49 \mathrm{eV}$, respectively. The relatively high barrier associated with the latter jumps makes the particles practically impenetrable for Co. This asymmetric minimum energy path for Co diffusion is due to the varying nature of the $\mathrm{Co}-\mathrm{Sc}$ interaction as a function of separation between the two. In the neighborhood of the particles, inside of the stress-affected zone, $D_{\mathrm{p}} \neq 0$, and therefore a certain diffusion retardation can also be expected (Fig. 5).

\section{Elastic field around a precipitate}

The stress field caused by the $\mathrm{Al}_{3} \mathrm{Sc}$ precipitates in the matrix is calculated according to Eshelby's solutions for spherical inclusions in an infinite isotropic and elastic body [60]. The radial dependencies for the strain $\epsilon_{r}$ and stress $\sigma_{r}$ fields are

$$
\begin{aligned}
\epsilon_{r} & =\frac{1+v}{3(1-v)}\left(\frac{R}{r}\right)^{3}(\Delta a+\Delta \gamma \Delta T) \text { and } \\
\sigma_{r} & =E \epsilon_{r} .
\end{aligned}
$$

Here, $v$ is the Poisson ratio of the matrix, $R$ is the precipitate radius, $r$ the radial distance from the precipitate center, $\Delta a$ the lattice misfit between the precipitate and the matrix, $E$ the Young modulus of the matrix, and $\Delta \gamma$ is the difference of the thermal expansion coefficients between the precipitate and the matrix. We have taken into account that the precipitates are formed and relaxed during hot deformation or annealing, while diffusion measurements are performed at nearly room temperature $(320 \mathrm{~K})$ which corresponds to the temperature difference $\Delta T$ in Eq. (5). The value of $\Delta \gamma$ is taken from DFT calculations [61] which were performed for both pure Al and $\mathrm{Al}_{3} \mathrm{Sc}$, including all relevant finite-temperature excitation mechanisms. The electronic and anharmonic contributions to $\Delta \gamma$ were found to be negligible at $320 \mathrm{~K}$.

The elastic strains (compressive as the $\mathrm{Al}_{3} \mathrm{Sc}$ precipitate adapts to the smaller lattice constant of the Al matrix) are maximum at the particle surface and decay with increased distance from the particle as $1 / r^{3}$. Typically, the strain becomes small $(\approx-0.01 \%)$ at three times the particle radius $(r=3 R)$, see Fig. 8. Elastic stresses behave qualitatively similarly, Eq. (6). Note that the yield stress of the present Al-based alloy is $305 \mathrm{MPa}$ [62].

In the case of vacancy-mediated diffusion, the diffusion coefficient of a solute, $D$, is generally $[33,34]$

$$
D=\frac{1}{6} f \lambda^{2} v_{0} \exp \left(-\frac{\Delta G^{f}+\Delta G^{b}+\Delta G^{m}}{k_{\mathrm{B}} T}\right) .
$$

Here $\lambda$ is the jump distance, $v_{0}$ the attempt (Debye) frequency typically on the order of $10^{12}-10^{13} \mathrm{~s}^{-1}, f$ the correlation factor, and $\Delta G^{f}, \Delta G^{b}$, and $\Delta G^{m}$ are the free energies of vacancy formation, solute-vacancy binding, and of vacancy migration, respectively. We emphasize that the present tracer diffusion experiments are performed under nearly equilibrium conditions, since the deformation-induced vacancies in the deformed Al matrix relax already at room temperature [63].

The correlation factor $f$ is temperature dependent for solute diffusion, since the frequencies of vacancy-atom exchanges depend on the type of atom and the distance from a solute atom [34]. It could be calculated in terms of the five-frequency model [64] for the fcc lattice but is considered as a constant (at the given temperature of the diffusion measurements) in the present work. Moreover, the effect of pressure on the total term $f \lambda^{2} v_{0}$ is neglected [34]. This is a reasonable assumption, since the strain dependencies in the exponent are more significant than in the prefactor. This finding can also be realized from the fact that the contribution of the pressure dependency of $f \lambda^{2} v_{0}$ to the vacancy formation volume is very small, around $1-3 \%$ of $\Omega$ [34].

\section{D. $A b$ initio energetics of Co diffusion in $\mathrm{Al}$}

Figure 6 shows the $a b$ initio calculated energy contributions entering the exponent in Eq. (7). They have initially been determined as Helmholtz energies for hydrostatic strain values up to $\pm 1.5 \%$. The maximum elastic strain of $1.5 \%$ corresponds to the elastic misfit between the pure Al matrix and $\mathrm{Al}_{3} \mathrm{Sc}$ particles, determined in Sec. $\mathrm{VA}$. By performing the calculations for $2 \times 2 \times 2$ and $3 \times 3 \times 3$ supercells we confirmed that finite-size errors at the maximum strain are below $5 \%$. The convergence is determined by the point defect interaction energies as a function of distance in the fcc $\mathrm{Al}$ matrix: While the Co-Co interaction energy is $155 \mathrm{meV}$ in a nearest-neighbor configuration, it is already in the fifth nearest neighbor shell decreased to $10 \%$ of this value. The results of $3 \times 3 \times 3$ supercells, plotted in Fig. 6, can therefore be considered as sufficiently accurate.

We observe that the vacancy formation energy, $E_{\text {form }}^{\mathrm{Vac}}$, the Co-vacancy binding energy, $E_{\text {bind }}^{\text {Co-Vac }}$, and the Co migration energy, $E_{\mathrm{m}}$, depend all almost linearly on the applied strain. The computed values at zero strain are $0.63,-0.02$, and $1.32 \mathrm{eV}$, respectively. The results agree well with previously measured values [65-68] varying between $0.62-0.71 \mathrm{eV}$ for $E_{\text {form }}^{\text {Vac }}$. Our computed values are also in a reasonable agreement with previously calculated values of $1.18 \mathrm{eV}$ for $E_{\mathrm{m}}$ [69] and -0.01 and $-0.04 \mathrm{eV}$ for $E_{\text {bind }}^{\text {Co-Vac }}$ [70-72]. The term $E_{\text {form }}^{\mathrm{Vac}}(p)$ has been determined from defect and defect-free structures that are evaluated at the same pressure, using pure fcc $\mathrm{Al}$ as a reference system for the pressure (upper $x$ axis) corresponding to a certain strain state (lower $x$ axis). The Legendre transformation from Helmholtz to Gibbs energies further requires the addition of the pressure-volume work $p \Omega_{\mathrm{v}}$ with the vacancy formation volume

$$
\Omega_{\mathrm{v}}=V(N-1, p)-\frac{N-1}{N} V(N, p),
$$

where $V(N, p)$ is the volume of a supercell with $N$ atoms at pressure $p$. The largely strain-independent term $E_{\text {form }}^{\mathrm{Vac}}(p)$ justifies the experimental approach, to identify $\Delta G_{f}$ in Eq. (7) with $p \Omega_{\mathrm{v}}$. As an estimate, the value of the vacancy activation volume measured for $\mathrm{Zn}$ GB diffusion in $\mathrm{Al}$ [35], $\Omega_{\mathrm{v}}=0.8 \Omega$, can be used for Co GB diffusion in Al.

The strain dependence of the migration barrier energy cannot be directly obtained from experiment and we therefore use again the result from DFT calculations for the present evaluation. The full diffusion profile for the different strain states is shown in Fig. 6(b). The respective initial state corresponds to the Gibbs energy of a vacancy formation next to a Co atom as given by the sum $E_{\mathrm{form}}^{\mathrm{Vac}}(p)+p \Omega_{\mathrm{v}}+$ $E_{\text {bind }}^{\text {Co-Vac }}$. In this configuration, Co still has a higher coordination 


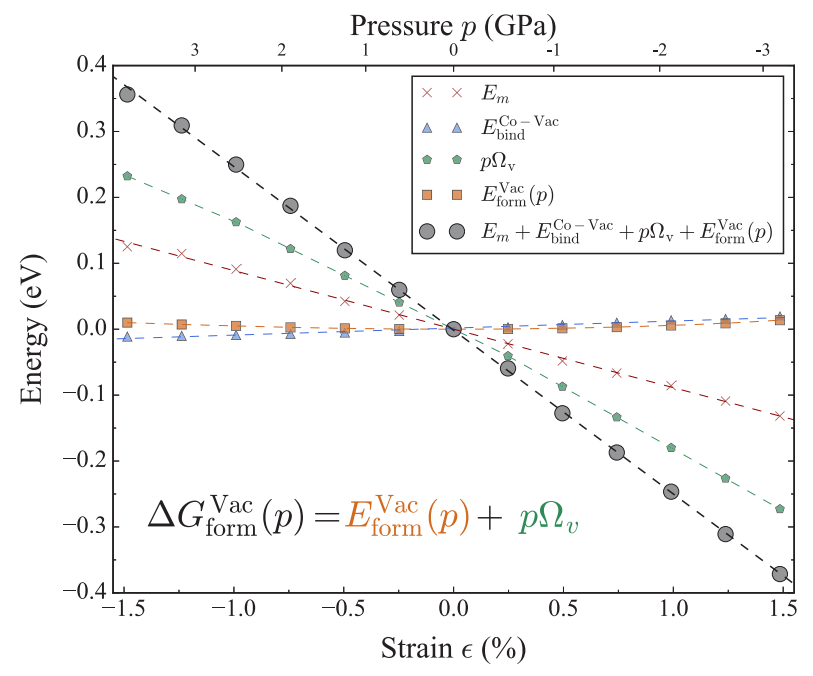

(a)

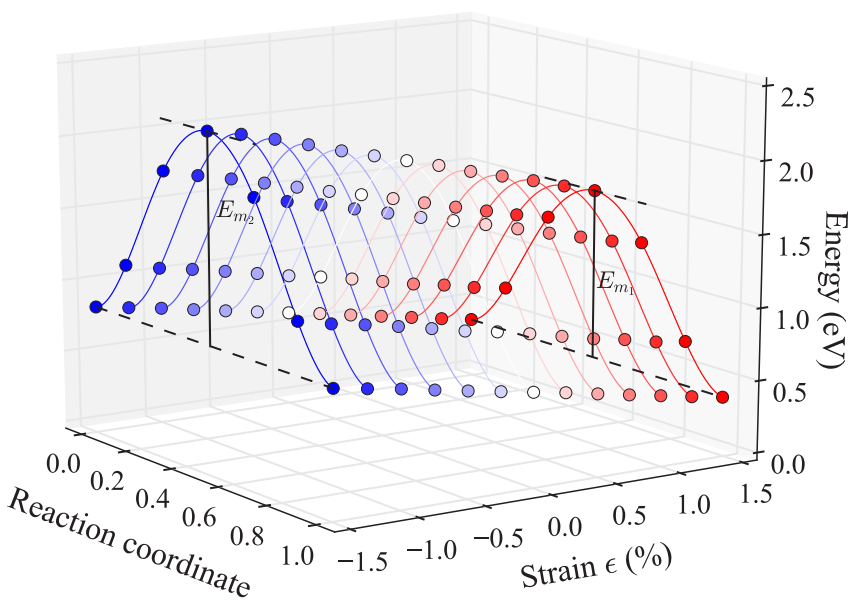

(b)

FIG. 6. (a) Strain dependence of the vacancy formation energy $E_{\mathrm{form}}^{\mathrm{Vac}}$, the Co-vacancy binding energy $E_{\mathrm{bind}}^{\mathrm{Co} \text {-Vac }}$, and the Co migration energy $E_{\mathrm{m}}$ for vacancy mediated nearest neighbor jumps in pure Al. In addition the pressure-volume work $p \Omega_{\mathrm{v}}$ is provided, which also enters the sum of all energy contributions. All the curves have been rescaled with respect to their respective energy value corresponding to $\epsilon=0$ (see text). (b) 3D representation of the variation of the energy profile (for Co atom diffusing into the nearest neighbor vacancy) along the minimum energy path for different strain rates. The respective initial state corresponds to the Gibbs energy of a vacancy formation next to a Co atom.

(11-fold) than in the saddle point configuration. This could explain why the latter is comparatively more sensitive to a compressive/dilatational straining of the lattice [see Fig. 6(b)]. Nevertheless, also the migration barrier of Co in Al follows to a good approximation a linear dependence on the applied strain, for which the expression

$$
E_{m}=(-0.09 \epsilon+1.31) \mathrm{eV}
$$

will be used in subsequent estimates. The strain dependence of $E_{m}$ is obtained using hydrostatically strained bulk supercells. For simplicity, we identify the radial strain dependence from Eshelby's solution with a hydrostatic strain and the corresponding computed migration barrier values from Eq. (9) are used to evaluate the diffusion constant away from the particle.

The calculations further reveal that the maximum change in the Co-vacancy binding energy due to the strain is $0.015 \mathrm{eV}$ if $|\epsilon| \leqslant 1.5 \%$. This is by an order of magnitude smaller than the change of the migration barrier $(0.12 \mathrm{eV})$. Therefore, the change of the GB diffusion coefficient of the tracer atom Co due to applied elastic stresses is estimated as

$$
\frac{D_{\mathrm{gb}}^{\mathrm{Co}}(\sigma)}{D_{\mathrm{gb}}^{\mathrm{Co}}(0)}=\exp \left(\frac{E_{m}(0)-E_{m}(\sigma)}{k_{\mathrm{B}} T}\right) \exp \left(-\frac{\frac{1}{3} \sigma_{i i} \Omega_{\mathrm{v}}}{k_{\mathrm{B}} T}\right) .
$$

Considering the impact of strain on the diffusion energetics, Figs. 2(b) and 2(c) plot the variation of effective diffusivity with the particle size and the particle fraction for two limiting cases with the enrichment factor $k_{s}=1$ and 1000 , respectively. The observations highlight the qualitative impact of Co segregation close to the interface which is quantified by $k_{s}$ in our diffusion model (see Appendix A). The radial dependence of effective diffusivity is mainly determined by the Eshelby strain dependence [Eq. (5)] and the resulting migration barriers. The differences between Figs. 2(b) and 2(c), however, indicate that a strong segregation as a driving force is required to make the Eshelby effect relevant. Accordingly, the radial strain dependence will not become noticeable [Fig. 2(b), $\left.k_{s}=1\right]$ unless the Co atoms are forced to approach the particle interface. Hence, we can indirectly conclude from the experiments that a strong Co segregation close to the particles within the grain boundaries exists.

In order to determine the enrichment factor in Eq. (4), we have also determined the segregation energy of $\mathrm{Co}$ as a function of biaxial strain at the (100) coherent $\mathrm{Al} / \mathrm{Al}_{3} \mathrm{Sc}$ interface and distance (in terms of layers) from the interface towards the Al matrix. DFT calculations performed for a precipitate in bulk reveal a complex segregation energy landscape (Fig. 7). The segregation energy at the interface is positive, indicating no enrichment or antisegregation. Our findings indicate a tendency for Co to preferentially segregate in the vicinity of

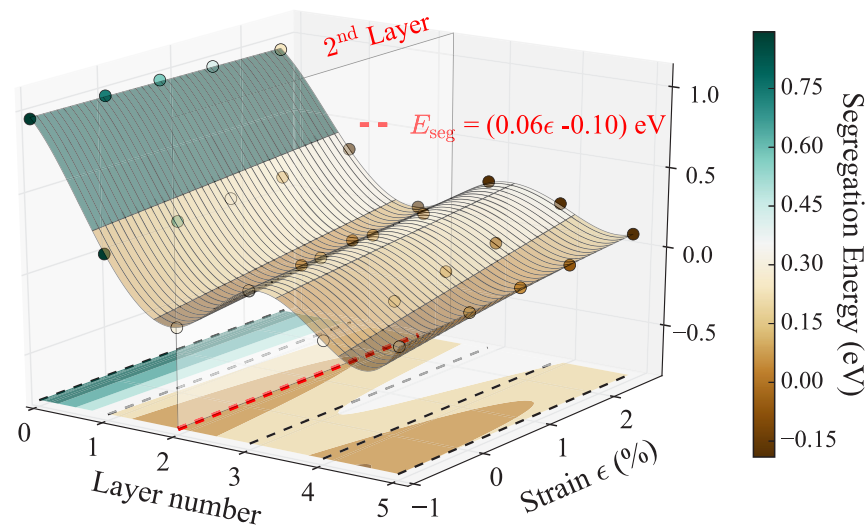

FIG. 7. Variation of Co segregation energy $E_{\mathrm{seg}}$ as a function of biaxial interfacial strain and layers away from the $\mathrm{Al} / \mathrm{Al}_{3} \mathrm{Sc}$ interface into the Al matrix. Negative values of the segregation energies represent favorable segregation and vice versa. The circles on the surface represent the $a b$ initio computed $E_{\text {seg }}$ values [Eq. (2)]. 
the $\mathrm{Al} / \mathrm{Al}_{3} \mathrm{Sc}$ interface two layers (oriented along the [100] direction) away into the matrix phase under tensile biaxial strains. For instance, at roughly $-1 \%$ strain, the segregation energy at the second layer is approximately $-0.16 \mathrm{eV}$, while it is $0.95 \mathrm{eV}$ (that in fact corresponds to desegregation) at the interface. Note that the finite segregation energies even at large distances (fifth layer) are combinedly due to the applied biaxial strains in the slab calculations, which does not decay with distance, and the strain-free reference value in Eq. (2).

At room temperature, the computed misfit strain between $\mathrm{Al}$ and $\mathrm{Al}_{3} \mathrm{Sc}$ is $1.2 \%$. The value of $E_{\text {seg }}$ at the favorable site (two layers away from the interface, Fig. 7) for 1.2\% strain is about $-0.02 \mathrm{eV}$. Although this value indicates that qualitatively we do have Co segregation, quantitatively it results in a very small enrichment [closer to $k_{s}=1$ than to $k_{s}=1000$, Fig. 2(b)] and a corresponding overestimation of measured values. The experimentally observed nonmonotonous variation of effective diffusivity along grain boundaries, however, is well captured [Fig. 2(c)] by choosing $k_{s}=1000$. This value translates into a segregation energy of $-0.2 \mathrm{eV}$ which is an order of magnitude larger than the value obtained from our bulk calculations. Among other possible reasons, this difference could be due to the fact that our calculations have been performed for a precipitate embedded in bulk whereas the diffusivity was measured along general high-angle grain boundaries. For this scenario, segregation between the particle and the grain boundary - in fact the triple line segregationbecomes relevant. Marquis et al. [73] observed a similarly strong preferential behavior for $\mathrm{Mg}$ segregation in the vicinity of the $\mathrm{Al} / \mathrm{Al}_{3} \mathrm{Sc}$ interface consistent with the $\mathrm{Mg}$ enrichment in the matrix phase slightly away from the interface as observed by atom-probe microscopy.

\section{E. General multishell Maxwell-Garnett (MMG) model of the particle effect on GB diffusion}

As mentioned above, our investigation is focused on the small, coherent $\mathrm{Al}_{3} \mathrm{Sc}$ precipitates, whose fraction at grain boundaries is estimated to be about $0.2 \%$. The larger, noncoherent, sparse $\mathrm{Al}_{6} \mathrm{Mn}$ particles are not taken into account. The net effect of the $\mathrm{Al}_{3}$ Sc particles on Co GB diffusion was estimated using the DFT-based information according to the following scheme:

(1) The elastic strain and stress fields around the coherent precipitates are determined, Eqs. (5) and (6).

(2) The jump barriers, $E_{m}(\sigma)$, at the given radial positions are determined, Eq. (9).

(3) The pressure-volume work term $\sigma \Omega_{\mathrm{v}}$ is determined from Eq. (6) using $\Omega_{\mathrm{v}}=0.8 \Omega$ for grain boundaries.

(4) The resulting variation of the GB diffusion coefficient of Co atoms is estimated by Eq. (10) as a function of the radial distance from the precipitates.

Figure 8 illustrates these calculations for the case of a coherent $\mathrm{Al}_{3} \mathrm{Sc}$ precipitate of the size of $20 \mathrm{~nm}$ as a function of the distance from the precipitate center. The determined elastic strain is plotted by blue circles (left ordinate), the corresponding change of the migration barrier $\Delta E_{m}(\sigma)$ and the $\sigma \Omega_{\mathrm{v}}$ term are shown by up and down triangles, respectively (right ordinate), and the estimated variation of the Co GB diffusion coefficient (normalized by the strain-free case) is

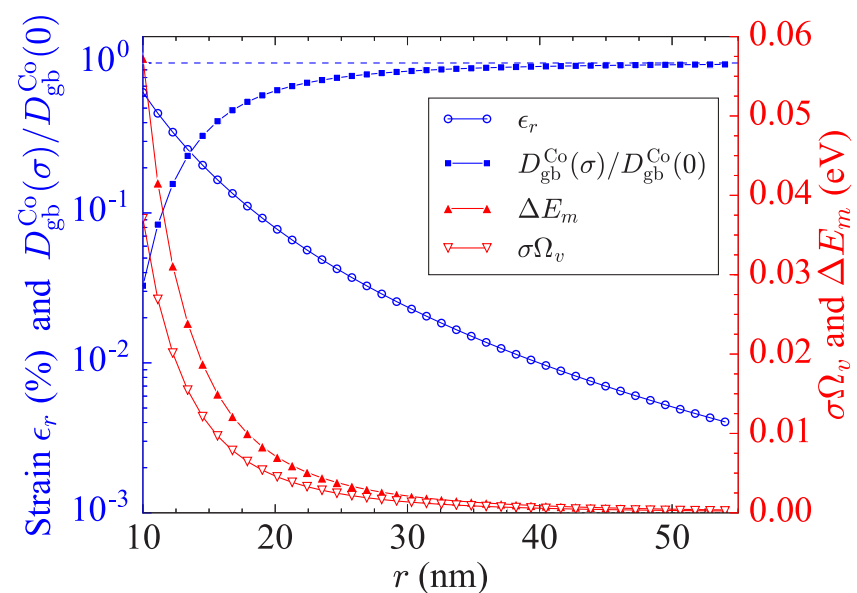

FIG. 8. The diffusion retardation, $D_{\mathrm{gb}}^{\mathrm{Co}}(\sigma) / D_{\mathrm{gb}}^{\mathrm{Co}}(0)$, (blue squares, left ordinate) as a function of radial distance $r$ from the particle center plotted together with the radial dependencies of the elastic strain $\epsilon$ (blue circles, left ordinate), the calculated change of the migration barrier $\Delta E_{m}$ (red triangles up, right ordinate), and the pressurevolume work term $\sigma \cdot \Omega_{\mathrm{v}}$ (red triangles down, right ordinate). The $20 \mathrm{~nm}$ large coherent $\mathrm{Al}_{3} \mathrm{Sc}$ particle is considered which is why the starting $R$ value is $10 \mathrm{~nm}$, representing the edge of the particle.

given by blue squares (left ordinate). The latter is normalized by the strain-free case, for which we used the measurement of the as-cast state. It drops by orders of magnitude at the particle edge but approaches its stress-free value at a distance of about three times the radius [Fig. 8].

The thus obtained strain/stress induced diffusion retardation, $D_{\mathrm{gb}}^{\mathrm{Co}}(\sigma) / D_{\mathrm{gb}}^{\mathrm{Co}}(0)$, is used in Eq. (4) to evaluate the net effect on the long-range effective Co GB diffusion coefficient. This equation captures in addition the impact of the particle size and density, as described by the particle fraction $f_{\mathrm{p}}$ and the segregation of $\mathrm{Co}$ atoms at the $\mathrm{Al} / \mathrm{Al}_{3} \mathrm{Sc}$ triple lines, as described by the enrichment factor $k$.

In the case of coherent particles the strain fields around the particles are taken into account in an approximate and iterative way. Using the spherical assumption [58] underlying Eq. (4), we divide the GB area around a precipitate by a number of concentric spheres (example shown in Fig. 9). The region inside the particle (including the interface of thickness $\delta_{s}=$ $0.5 \mathrm{~nm}$ ) is assumed to be impermeable for diffusing atoms. Then Eq. (4) is sequentially used to estimate the effective diffusivities within subsequent regions, taking the effective diffusivity of the previous sphere/shell as $D_{\mathrm{p}}$ for the next one.

A sufficiently large cutoff radius has to be chosen for the outermost shell to include the whole elastic contribution. For the present investigation, a value of $3.5 R$ was found to give converged results below $1 \%$ for the complete experimental range of particle fractions. This can be easily understood from Fig. 8, where the effective diffusivity almost achieves its stress-free value at a distance $3.5 R$ from the interface. The procedure requires a proper re-scaling of the particle volume fractions $f_{\mathrm{p}}$ and the enrichment factors $k$. The latter represent the ratio of averaged tracer concentrations within the particle and the remaining matrix [58]. Our multishell approach of solving the Maxwell-Garnett equation takes care of the fact that the real segregation of Co atoms at the particle/matrix 
interface is effectively spread over sequentially larger effective 'particles' of radii $m_{x} R, x=1 \ldots n$ where $x$ denotes a shell with radius $m_{x} R\left(m_{x} \in \mathbb{R}\right)$ and $n$ is the total number of shells around the particle. This is to account for a solute enrichment in an inner shell with respect to the next larger one, see Appendix A.

Even for large semicoherent particles, the coherency strain does not vanish completely. Instead, the introduction of a misfit dislocation at the interface is accompanied by residual coherency strain field around the particles. For the model predictions in Fig. 2(c) (for $R>20 \mathrm{~nm}$ ), the strain field is determined based on the square lattice approach [74,75]. More details of the model and a representative example are provided in Appendices $\mathrm{A}$ and $\mathrm{C}$.

The last value of the iteration (sphere of radius $m_{n} R$ ) is used for the final calculation of the effective diffusivity of a GB plotted in Fig. 2(c). We underline that we are not expecting a full agreement of the model predictions with the experimental data. While the DFT computations used for the present model were performed for an $\mathrm{Al}_{3} \mathrm{Sc}$ particle embedded in pure Al matrix, the experimental diffusion occurs along general high-angle grain boundaries. Nevertheless, the similar trends between theory and experiment in Fig. 2(c) confirm the validity of our hypothesis.

The particle growth induced by the thermal annealing treatments at 723 and $773 \mathrm{~K}$ explains the decrease of the effective diffusion coefficient if the particles are still coherent. It is the state in which the GB diffusion coefficient of Co atoms is slowest and amounts to about $50 \%$ of the value in the ECAP-processed state. As a result of further annealing at higher temperatures, the precipitates grow above the critical size and loose their coherency with the matrix. Simultaneously, there is a dissolution of the particles and a decrease of the corresponding fraction. Therefore, the Co GB diffusion coefficient increases approaching the values typical for the precipitate-free $\mathrm{Al}$ alloy [Fig. 2(c)].

\section{ADDITIONAL EFFECTS}

\section{A. Effect of segregation on GB diffusion}

The faster diffusion in pure Al might indicate an alloying effect on GB diffusion. It is a general trend observed experimentally that the purer the material, the higher is the GB diffusion rate, as it was found, e.g., for GB self-diffusion in pure $\mathrm{Cu}$ [76] or $\mathrm{Ni}$ [44,77] or solute (Ni) GB diffusion in pure $\mathrm{Cu}$ [78]. Due to their large size, $\mathrm{Mg}$ atoms are known to segregate to GBs in the present alloy after annealing at slightly elevated temperatures [7]. This has also been observed in other Al-Mg alloys [79]. The Mg atoms occupy the sites otherwise available for Co diffusion, eventually leading to a reduced GB diffusion with respect to that in pure Al, Table I. Moreover, $\mathrm{GB}$ reconstruction in the $\mathrm{Al}$ alloy (following, e.g., the GB complexion theory [80]) might be responsible, too. We have not observed any direct indications of hypothetical GB reconstructions in the current experiments. However, experimental hints of the existence of such temperature-induced interface reconstructions have been provided by measuring $\mathrm{Ag}$ grain boundary diffusion in $\mathrm{Cu}$ near $\Sigma 5$ grain boundaries [81,82]. Careful atomistic modeling revealed and provided a full characterization of temperature-induced [83] and segregationinduced [84] interface transformations.

In the case of the ECAP processing at $573 \mathrm{~K}$ and post annealing at higher temperatures, however, the chemical analysis did not reveal any measurable segregation at most of the GBs (about 80\%) in the limits of the uncertainty of the EDX measurements [7]. In some of the GBs, a slight increase of the local $\mathrm{Mg}$ concentration by about $0.5 \%$ could be detected. Still, a significant increase of the $\mathrm{Mg}$ concentration was observed at triple junctions. Following the above reasonings, an increase of the Co GB diffusion rate may be expected in the ECAP-processed state with respect to that in the as-cast state, though a decrease is seen, Table I. Thus, a possible segregation of $\mathrm{Mg}$ in the as-cast state and its reduction with the hot processing/annealing does not explain the experimental trends for Co diffusion.

One may have a certain grain boundary (GB) diffusion of $\mathrm{Al}, \mathrm{Sc}$, and $\mathrm{Zr}$ atoms during the present $\mathrm{GB}$ diffusion measurements too. Using DFT-based calculations, however, both $\mathrm{Zr}$ and $\mathrm{Sc}$ are found to exhibit a strongly repulsive interaction with $\mathrm{Co}$ on the first two nearest neighbor sites $\left[E_{\mathrm{int}}^{\mathrm{Co} Z \mathrm{Zr}}: 167.2,105.2 \mathrm{meV}\right.$, respectively; $E_{\mathrm{int}}^{\mathrm{Co}-\mathrm{Sc}}: 133.8,89.6$ $\mathrm{meV}$, respectively] with a slightly attractive interaction on the third [ $\left.E_{\text {int }}^{\mathrm{Co}-\mathrm{Zr}}:-37.8 \mathrm{meV} ; E_{\mathrm{int}}^{\mathrm{Co}-\mathrm{Sc}}:-31.6 \mathrm{meV}\right]$. Moreover, no $\mathrm{GB}$ segregation of $\mathrm{Sc}$ and $\mathrm{Zr}$ was observed in our experiments, which would have otherwise affected the Co GB diffusion by occupying the potential diffusion sites for Co atoms.

\section{B. Effect of SPD processing on GB diffusion}

The present results substantiate that the severe plastic deformation of the Al-based alloy, in contrast to the ECAP processing of $\mathrm{Ni}$ or $\mathrm{Ti}$, does not produce a deformation-modified state of the grain boundaries which would be characterized by enhanced diffusion coefficients. On one hand, a decrease of the GB diffusion coefficients as a result of the ECAP processing was already observed for the case of Co diffusion in ultrafine grained Ti. However this represents a very special case in view of the diffusion mechanism of Co atoms in the hexagonal closepacked (hcp) lattice of $\alpha$-Ti [15]. On the other hand, vacancymediated GB diffusion of $\mathrm{Ag}$ atoms was extremely enhanced in ECAP-processed $\alpha$ - Ti [15]. These facts correspond to the generation of excess free volume at deformation-modified GBs, which serve as traps for interstitially diffusing Co and as vehicles for substitutionally diffusing Ag atoms. Since the substitutional diffusion mechanism holds definitely for Co in Al [8], one may safely assume substitutional solubility and a vacancy-mediated diffusion mechanism for Co atoms in grain boundaries of $\mathrm{Al}$ and $\mathrm{Al}$ alloys, too.

The absence of an enhancement of Co GB diffusion after SPD processing of Al-based alloy is most probably related to a relatively high homologous temperature of the deformation, $T_{\text {def }} / T_{m}$, and need a clarification (here $T_{m}$ is the corresponding melting temperature). Note that $T_{\mathrm{def}} / T_{m}$ is about 0.6 in the present case.

\section{Relaxation of the deformation-modified state}

Nazarov and co-workers [22] described a deformationmodified ('nonequilibrium') state of GBs in terms of arrays of extrinsic GB dislocations. These defects were proposed to relax 
by dislocation climb and annihilation at elevated temperatures $T$. The corresponding relaxation time $\tau$ was estimated as [85]

$$
\tau=\frac{k_{\mathrm{B}} T d^{3}}{A \delta D_{\mathrm{gb}}^{s d} G \Omega}
$$

where $k_{\mathrm{B}}$ is the Boltzmann constant, $d$ the average grain size, $\delta$ the GB width, $D_{\mathrm{gb}}^{s d}$ the self-diffusion coefficient for general relaxed high-angle GBs, $G$ the shear modulus, $\Omega$ the atomic volume, and $A$ a geometrical factor. The value of $A$ was suggested to lie between 100 and 500, depending on the specific disclination model of the ultrafine grained materials used [85], and it was shown that this value gives reasonable estimates for SPD-processed $\mathrm{Ni}$ [14]. As stated above, the diffusional GB width $\delta \approx 0.5 \mathrm{~nm}$ [44]. The GB self-diffusion coefficient in Al, $D_{\mathrm{gb}}^{s d}$, is estimated to be about $2.8 \times 10^{-16} \mathrm{~m}^{2} / \mathrm{s}$ at $320 \mathrm{~K}$ based on the Arrhenius parameters reported in Ref. [86]. Using the material parameters of $\mathrm{Al}\left(G=26 \mathrm{GPa}\right.$ and $\left.\Omega=16.6 \AA^{3}\right)$, the relaxation time of the deformation-modified state of the GBs in $\mathrm{Al}$ is on the order of seconds at the measurement temperature of $320 \mathrm{~K}$. Taking a finite cooling time for a deformed billet from the deformation $(573 \mathrm{~K})$ to the room temperature into account, one may safely conclude that a hypothetical enhancement of the diffusion rates due to a deformation-modified state of GBs, if it does exist during deformation, relaxes and cannot be measured in the present post-mortem experiments.

This conclusion fully agrees with the present results on GB diffusion of Co after ECAP processing, since we do not observe any enhancement of the diffusion rates in the deformationmodified state. However, the decrease of the Co GB diffusion rate after ECAP processing is not explained by this effect.

\section{Effect of crystal dislocations}

The typical dislocation density in a well-annealed metallic material [34] is on the order of $10^{10} \mathrm{~m}^{-2}$. In contrast to this, in addition to the excess GB dislocations and the GB modifications, SPD processing is known to induce a high density of crystal dislocations, which may approach the values on the order of $10^{15} \mathrm{~m}^{-2}$ for $\mathrm{Ni}$ or $\mathrm{Cu}$ [87] and $10^{14} \mathrm{~m}^{-2}$ for Al alloys [88]. Due to the associated higher atomic jump rates compared to in the bulk, dislocations in metals are known to represent generally short-circuit diffusion paths [33,34] and may modify diffusion transport during measurements of GB diffusion [89]. Note that deformation-induced vacancies annihilate below room temperature in plastically deformed Al [63] and, therefore, cannot affect the present diffusion measurements. In order to study the effect of dislocations on diffusion, at least two kinds of models have to be analyzed:

Model 1: Dislocation-enhanced diffusion. Since the Ctype conditions are fulfilled in the present diffusion experiments, the crystal dislocations represent diffusion short circuits and provide a diffusion enhancement with respect to bulk diffusion in addition to grain boundaries. Due to the associated low activation enthalpy as compared to that in bulk, the dislocation diffusion can be significant at low temperatures [34]. However, the rates of dislocation diffusion correspond typically to about $1 / 10$ th of the diffusion rate along general high-angle GBs $[33,90]$ and, thus, the dislocation diffusion contribution should not be observed in the present experiments apart from several first near-surface points of the concentration profiles in Fig. 1.

Although the dislocation density is larger in ECAPprocessed material by orders of magnitude, GB diffusion is slower in the ultrafine grained state with respect to that in pure Al. ECAP processing does not enhance Co GB diffusion in spite of a largely increased dislocation density with respect to the as-cast state. Alternatively, the post-deformation annealing treatment at, e.g., $823 \mathrm{~K}$ for $50 \mathrm{~h}$ enhances $\mathrm{GB}$ diffusion, while a dramatic decrease of the dislocation density is observed and therefore opposite to what would be expected within this model. Based on this, we conclude that Model 1 is not applicable to the present case.

Model 2: Dislocation-modified GB diffusion. The crystalline dislocations are attached not only to the free surface of the sample, but they cross the GBs, too. In the latter case, these dislocations represent the paths for enhanced leakage of the tracer atoms from grain boundaries. In the present case with the absence of bulk diffusion, the GB diffusion problem with out-diffusion via dislocation corresponds to the so-called C-B-type regime after Divinski et al. [91,92] (where the 'quasi' B-type regime corresponds to diffusion along the GBs with a tracer leakage via dislocations and the C-type regime corresponds to diffusion along the dislocations with no leakage to the crystalline bulk) or $\mathrm{D}_{1}$-type regime after Klinger and Rabkin [89,93]. The characteristic penetration profile in this regime is a sum of two contributions described as $\ln \bar{c} \propto y^{2}$ (C type) and as $\ln \bar{c} \propto y^{6 / 5}$ (B type) [33]. The tracer leakage from GBs is controlled by the parameter $\alpha^{*}$, which is [compare with Eq. (3)]

$$
\alpha^{*}=\frac{s \delta}{2 g_{\mathrm{d}} \sqrt{D_{\mathrm{d}} t}} .
$$

Here $g_{\mathrm{d}}=A_{\mathrm{d}} \rho_{\mathrm{d}}$ is the volume fraction of sites belonging to the dislocation pipes, with $A_{\mathrm{d}}$ being the cross section of the dislocation pipe and $\rho_{\mathrm{d}}$ the dislocation density. In the case of $\alpha^{*}>1$, the C-type regime continues to hold and Eq. (1) has to be applied to determine the corresponding GB diffusion coefficients (from the slope of $\ln \bar{c}$ versus $y^{2}$ ). The situation changes if $\alpha^{*}<0.1$ (and $\alpha$ remains to be large, $\alpha>1$ ) [89], in which case the concentration profiles have to be analyzed in the coordinates of the logarithm of concentration vs the penetration depth $y$ (i.e., for the quasi B-type conditions, where the word 'quasi' corresponds to a B-type like regime because the out-diffusion of tracer occurs only at the dislocation/GB crossing [33]) and the only quantity that can be determined is the modified triple product

$$
P=s^{*} \cdot \delta \cdot D_{\mathrm{gb}}=1.128 \sqrt{\frac{D_{\mathrm{eff}}}{t}}\left(-\frac{\partial \ln \bar{c}}{\partial y}\right)^{-2}
$$

instead of the diffusion coefficient $D_{\mathrm{gb}}$ [89]. Here,

$$
D_{\text {eff }}=g_{\mathrm{d}}^{2} D_{\mathrm{d}}
$$

is the effective diffusivity, which describes the tracer leakage from GBs into the crystal volume by dislocation pipe diffusion. In Eq. (13), $s^{*}$ is the segregation coefficient for the tracer atoms between the GBs and the dislocation pipes which is probably a value of the order of unity. Estimating the diffusion coefficient 
along the dislocation pipes as $D_{\mathrm{d}}=1 / 10 D_{\mathrm{gb}}$ [33], the value of the parameter $\alpha^{*}$ can be determined (Table I).

Our analysis reveals that $\alpha^{*}>1$ for most of the processing conditions considered in this work and, therefore, the effect of dislocations can safely be neglected, thereby confirming that Eq. (1) was correctly used. The only result of the diffusion measurements for Co GB diffusion in ECAP-processed Al alloy with the highest dislocation density $\left(7 \times 10^{13} \mathrm{~m}^{-2}\right.$ without annealing) has to be revisited, since the corresponding value of $\alpha^{*}$ is less than unity (Table I). From the GB diffusion theory [33], it is well known that GB diffusion under conditions of $0.1<\alpha<1$ corresponds to a transition regime between the B- and C-type kinetics and the determined diffusion coefficients underestimate the real values [94]. Following the approach suggested in Ref. [94] and outlined in Ref. [33], a correction factor is determined and the corrected value of the corresponding diffusion coefficient is listed in Table I.

We conclude that these are the dislocations which effectively retard GB diffusion in the ECAP-processed state providing a tracer leakage from the GBs. Nevertheless, the corrected value is still lower in the ECAP-processed state with respect to that in the as-cast state.

\section{CONCLUSIONS}

Grain boundary diffusion of Co in an Al-based alloy is found to be slower than in pure $\mathrm{Al}$ and is not enhanced by severe plastic deformation. The absence of SPD-induced diffusion enhancement is explained by a fast relaxation of the deformation-modified state already at room temperature.

The deformation-induced dislocations do not practically affect GB diffusion in the ECAP-processed Al-based alloy. A post-deformation annealing treatment of the ultrafine grained $\mathrm{Al}$ alloy results in a nonmonotonous dependence of the $\mathrm{Co}$ GB diffusion rate on the annealing temperature, which first decreases up to a certain critical value beyond which it increases again. This behavior is explained by the evolution of the $\mathrm{Al}_{3} \mathrm{Sc}$ precipitates in the alloy, which experience coherency loss beyond a certain critical size.

An $a b$ initio informed phenomenological diffusion model is employed, which takes the strain fields around coherent $\mathrm{Al}_{3} \mathrm{Sc}$ precipitates into account. The effects of strains on the parameters affecting Co GB diffusion are determined by DFT calculations. The effective diffusion in the precipitated GBs is described as diffusion in a heterogeneous media with diffusionblocking precipitates. The importance of solute segregation to the precipitates is highlighted.

Extending the Maxwell-Garnett model, we iteratively solve for the effective diffusivity around the particles within a multishell approach taking DFT-based diffusion energetics and their explicit strain dependence into account. The presented multishell Maxwell-Garnett (MMG) model is general in its framework and therefore applicable to study similar phenomena in other alloy systems.

\section{ACKNOWLEDGMENTS}

Financial support from the Deutsche Forschungsgemeinschaft (DFG) within the priority program SPP-1713 "chemomechanics" (research projects HI 1300/8-2 and DI 1419/7-2) is gratefully acknowledged. The authors gratefully acknowledge the fruitful discussions on the square lattice model with Dr. S. Sampath and PD Dr. habil. R. Janisch.

\section{APPENDIX A: GB DIFFUSION MODEL}

The effect of particle size on the GB diffusion coefficient is evaluated employing the multishell Maxwell-Garnett equation in an iterative manner in concentric regions around the particle up to some cutoff radius. For demonstration, we will consider four regions-three concentric ones labeled 1, 2, 3 [Fig. 9(a)] of radii $r_{1}=1.5 R, r_{2}=2 R, r_{3}=3 R$ around the particle of radius $R\left(=r_{\mathrm{av}}\right)$ and the remaining area which are used to calculate the effective diffusivity in the presence of elastic

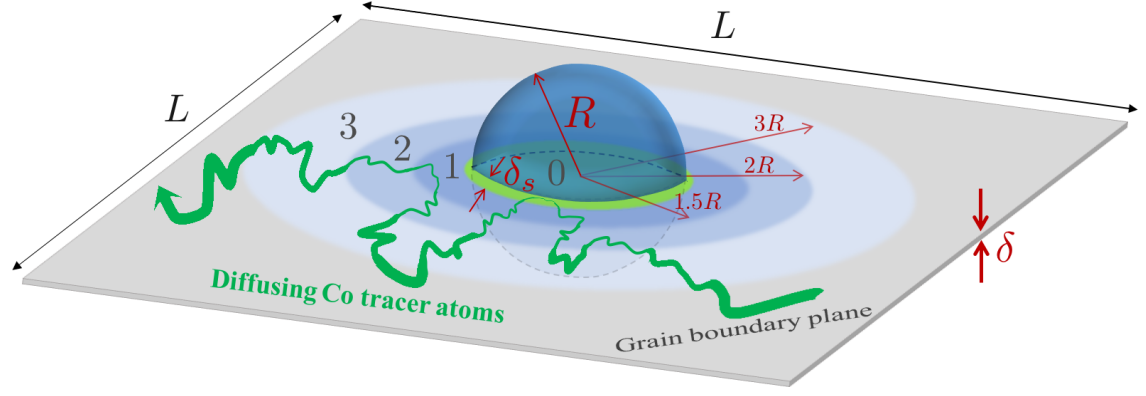

(a)

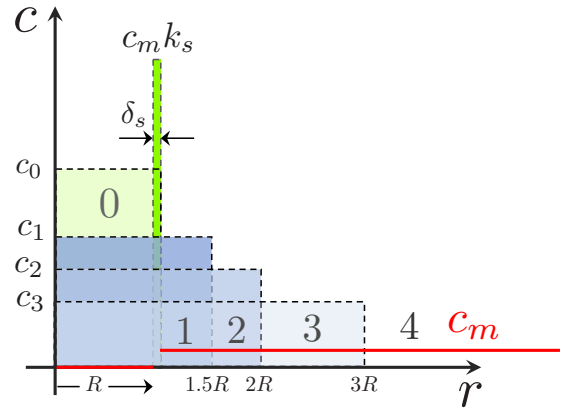

(b)

FIG. 9. Schematic illustration of the employed diffusion model taking the simple case of three concentric shells and $3 R$ as the cutoff radius. (a) A particle of radius $R$ (region 0 denoting $r \leqslant R+\delta_{s}$ ) present in the GB plane with three concentric regions of radii $1.5 R$ (region 1 ), 2R (region 2), and $3 R$ (region 3 ) used for calculating the effective diffusivity around it. The region labeled 4 denotes the unaffected GB area. The widths of the GB plane and the particle interface are denoted as $\delta$ and $\delta_{s}$, respectively, and are of similar magnitude. The green line sketches a continuous random diffusion path of a tracer Co atom within the GB plane. The line thickness in a given region of GB plane is schematically scaled with the corresponding Co effective diffusion coefficient in the particular region. (b) Estimation of the effective enrichment factors entering the expressions for the effective diffusivities for different regions considered in (a) around the particle. The horizontal solid red line for $r>R+\delta_{s}$ represent the tracer concentration in the matrix $c_{m}$ around the particle with the zero level inside the particle denoted by the smaller red line for $r<R$. The particle interface (width $\delta_{s}$ ) has a concentration $c_{m} k_{s}, k_{s}$ denoting the interfacial segregation factor. 
coherency strains around the particle present in the GB plane. The label 0 represents the region inside the particle $(r<$ $R+\delta_{s}$ ). The thin shell [colored green in Fig. 9(a)] of thickness $\delta_{s}(\ll R)$ around the particle represents the interface width where the Co tracer atoms experience interfacial segregation. The diffusivities $D_{\text {eff }}^{(i)}(i=1,2,3)$ of the concentric regions $r<$ $1.5 R, r<2 R$, and $r<3 R$ are estimated as effective values for two-phase materials composed of (effective) particles of the radius $R+\delta_{s}, 1.5 R, 2 R$ and the outer areas $R+\delta_{s}<$ $r<1.5 R, 1.5 R<r<2 R$, and $2 R<r<3 R$, respectively. According to Eq. (4), $D_{\text {eff }}^{(i)}$ are functions of the corresponding tracer enrichment factor $k_{i}$, effective particle volume fraction $f_{p}^{(i)}$, the effective GB diffusivity $D_{\mathrm{gb}}^{(i)}(\sigma)$ outside of the particle, and the effective diffusivity $D_{\text {eff }}^{(i-1)}$ within the particle.

$$
\begin{aligned}
& D_{\mathrm{eff}}^{(1)}=D_{\mathrm{eff}}\left(k_{1}, f_{p}^{(1)}, D_{p}=0\right) \times D_{\mathrm{gb}}^{(1)} \\
& D_{\mathrm{eff}}^{(2)}=D_{\mathrm{eff}}\left(k_{2}, f_{p}^{(2)}, D_{p}=D_{\mathrm{eff}}^{(1)}, D_{\mathrm{gb}}^{(2)}\right) \times D_{\mathrm{gb}}^{(2)} \\
& D_{\mathrm{eff}}^{(3)}=D_{\mathrm{eff}}\left(k_{3}, f_{p}^{(3)}, D_{p}=D_{\mathrm{eff}}^{(2)}, D_{\mathrm{gb}}^{(3)}\right) \times D_{\mathrm{gb}}^{(3)} \\
& D_{\mathrm{eff}}=D_{\mathrm{eff}}^{(4)}=D_{\mathrm{eff}}\left(k_{4}, f_{p}^{(4)}, D_{p}=D_{\mathrm{eff}}^{(3)}, D_{\mathrm{gb}}\right) \times D_{\mathrm{gb}},
\end{aligned}
$$

where the function $D_{\text {eff }}\left(k_{i}, f_{p}^{(i)}, D_{p}, D_{\mathrm{gb}}^{(i)}\right)$ represents the right hand side expression of the modified Maxwell-Garnett equation (4) for different regions and $D_{\mathrm{gb}}^{(i)}(\sigma)$ is computed using (10). The radial dependency of strain decay away from the particle is explicitly included in the calculation of $D_{\mathrm{gb}}^{(i)}(\sigma)$ via Eshelby's solution [Eq. (6)].

As an example, the effective diffusivity in the first shell (region 1) using (4) is written as

$$
\begin{aligned}
\frac{D_{\mathrm{eff}}^{(1)}}{D_{\mathrm{gb}}^{(1)}}=\frac{1}{1+f_{\mathrm{p}}^{(1)}\left(k_{1}-1\right)} \times \frac{\left(1-f_{\mathrm{p}}^{(1)}\right)+k_{1}\left(1+f_{\mathrm{p}}^{(1)}\right) \frac{D_{\mathrm{p}}}{D_{\mathrm{gb}}^{(1)}}}{\left(1+f_{\mathrm{p}}^{(1)}\right)+k_{1}\left(1-f_{\mathrm{p}}^{(1)}\right) \frac{D_{\mathrm{p}}}{D_{\mathrm{gb}}^{(1)}}} \\
\Rightarrow D_{\mathrm{eff}}^{(1)}=\frac{1}{1+f_{\mathrm{p}}^{(1)}\left(k_{1}-1\right)} \times \frac{\left(1-f_{\mathrm{p}}^{(1)}\right)}{\left(1+f_{\mathrm{p}}^{(1)}\right)} \times D_{\mathrm{gb}}^{(1)}, \quad, \quad(\mathrm{A} 2)
\end{aligned}
$$

assuming impenetrable particles $\left(D_{p}=0\right)$. While calculating the effective diffusivities $D_{\text {eff }}^{(i)}$ in regions 2,3 , and 4 , the effective diffusivity for the previous region $D_{\text {eff }}^{(i-1)}$ is taken as $D_{p}$ with the corresponding $f_{p}^{(i)}$ and $k_{i}$. The value of overall effective diffusivity $D_{\text {eff }}\left(=D_{\text {eff }}^{(4)}\right)$ in (A1) is calculated taking $D_{\mathrm{gb}}^{(4)}=D_{\mathrm{gb}}$, the reference value used while plotting the calculated curve $\left(D_{\text {eff }} / D_{\mathrm{gb}}\right)$ in Fig. 2(c).

\section{Determining the particle fractions $f_{p}^{(i)}$}

The effective particle volume fractions for each concentric shell are defined in terms of the area ratios (GB width $\delta \ll R$ ) of two adjacent shells as

$$
\begin{aligned}
f_{p}^{(1)} & =\frac{\pi R^{2}}{\pi r_{1}^{2}}, \quad f_{p}^{(2)}=\frac{\pi r_{1}^{2}}{\pi r_{2}^{2}}, \quad f_{p}^{(3)}=\frac{\pi r_{2}^{2}}{\pi r_{3}^{2}}, \quad f_{p}^{(4)}=9 x \\
\Rightarrow f_{p}^{(1)} & =\frac{4}{9}, \quad f_{p}^{(2)}=\frac{9}{16}, \quad f_{p}^{(3)}=\frac{4}{9}, \quad f_{p}^{(4)}=9 x, \quad(\text { A3 })
\end{aligned}
$$

where $x=\frac{\pi R^{2}}{L^{2}}$ is the volume fraction of the particle with $L^{2}$ being the area of GB plane assuming a square grain. The experimentally measured value of diffusivity in the stress-free state for the largest particle size $(60 \mathrm{~nm})$ is used as the reference value:

$$
D_{\text {eff }}^{\text {stress free }}=D_{\text {eff }}\left(k_{1}, f_{p}=x, D_{p}=0, D_{\mathrm{gb}}\right) \times D_{\mathrm{gb}} .
$$

\section{Determining tho Co tracer enrichment factors $\boldsymbol{k}_{\boldsymbol{i}}$}

Taking the tracer concentration $c_{m}$ in the matrix as reference, the Co enrichment factors $k_{i}$ within different regions $(i=1,2,3,4)$ around the particle are defined as

$$
k_{1}=\frac{c_{0}}{c_{m}}, \quad k_{2}=\frac{c_{1}}{c_{m}}, \quad k_{3}=\frac{c_{2}}{c_{m}}, \quad k_{4}=\frac{c_{3}}{c_{m}},
$$

where $c_{i}$ are the average tracer concentrations in different regions calculated as follows, see Fig. 9(b):

(i) The average concentration in region $0\left(r \leqslant R+\delta_{s}\right)$ is

$$
\begin{aligned}
c_{0} & =c_{m} k_{s} \frac{\left[\pi\left(R+\delta_{s}\right)^{2}-\pi R^{2}\right]}{\pi\left(R+\delta_{s}\right)^{2}}=c_{m} k_{s} \frac{\left[\pi \delta_{s}^{2}+2 \pi R \delta_{s}\right]}{\pi\left(R+\delta_{s}\right)^{2}} \\
& \approx c_{m} k_{s} \frac{2 \pi R \delta_{s}}{\pi R^{2}}=c_{m} k_{s} \frac{2 \delta_{s}}{R} \quad\left(\delta_{s} \ll R\right),
\end{aligned}
$$

where the term in the numerator, $c_{m} k_{s}\left[\pi\left(R+\delta_{s}\right)^{2}-\pi R^{2}\right] \approx$ $c_{m} k_{s} 2 \pi R \delta_{s}$, is the concentration in the concentric shell of thickness $\delta_{s}$ around the particle and $k_{s}$ is the Co segregation factor at the interface obtained as $k_{s}=\exp \left(-\frac{E_{\mathrm{seg}}}{k_{B} T}\right), E_{\text {seg }}$ being the $a b$ initio computed segregation energy of $\mathrm{Co}$ as a function of misfit strain in the vicinity of $\mathrm{Al} / \mathrm{Al}_{3} \mathrm{Sc}$ interface [Fig. 7]. The starting expression for $c_{0}$ can be understood as distributing the concentration in the concentric interface layer $(R<r<$ $R+\delta_{s}$ ) of width $\delta_{s}$ homogeneously within a circular region of radius $R+\delta_{s}$. The same idea also applies in the subsequent regions.

(ii) The general expression (for a model with $n$ shells) for the average concentration in any given shell (region $x, x=$ $1 \ldots n)$ with a radius of $m_{x} R$ can be written as

$$
c_{x}=\frac{c_{m} k_{s} 2 \pi R \delta_{s}+c_{m}\left[\pi\left(m_{x} R\right)^{2}-\pi\left(R+\delta_{s}\right)^{2}\right]}{\pi\left(m_{x} R\right)^{2}} .
$$

The generalized expression for the segregation factor $k_{x}$ in subsequent shells then becomes

$$
k_{x}=\frac{c_{x}}{c_{m}}=\frac{\left(m_{x}^{2}-1\right) R^{2}+2\left(k_{s}-1\right) R \delta_{s}-\delta_{s}^{2}}{m_{x}^{2} R^{2}} .
$$

The number of shells $n$ around the particles serves as a convergence parameter for refining the spacing between the shells while iteratively solving the above set of equations. For the current material system, 30 shells are found to be sufficient to provide accurate data with uncertainty well below $1 \%$. The outermost shell cutoff radius, however, has an upper bound which can be understood from Fig. 10. The multishell Maxwell-Garnett formalism yields the effective diffusivity assuming the particles are completely embedded in the matrix [Fig. 10(a)]. This requires $R_{\text {cut }} \leqslant \frac{L}{2}\left(=\frac{R}{2} \sqrt{\frac{\pi}{x}}\right)$, and $x$ is the volume fraction $\left(=\pi R^{2} / L^{2} ; \delta_{s} \ll R\right)$ if the precipitates are arranged on a simple square lattice. This expression can be adopted for any other regular arrangement. As $R_{\text {cut }}$ lies in the interval $(L / 2, L / \sqrt{2})$, the effective particles, i.e., the particles with respective shells around them, start overlapping [as shown in Fig. 10(b)] and the problem reverses 

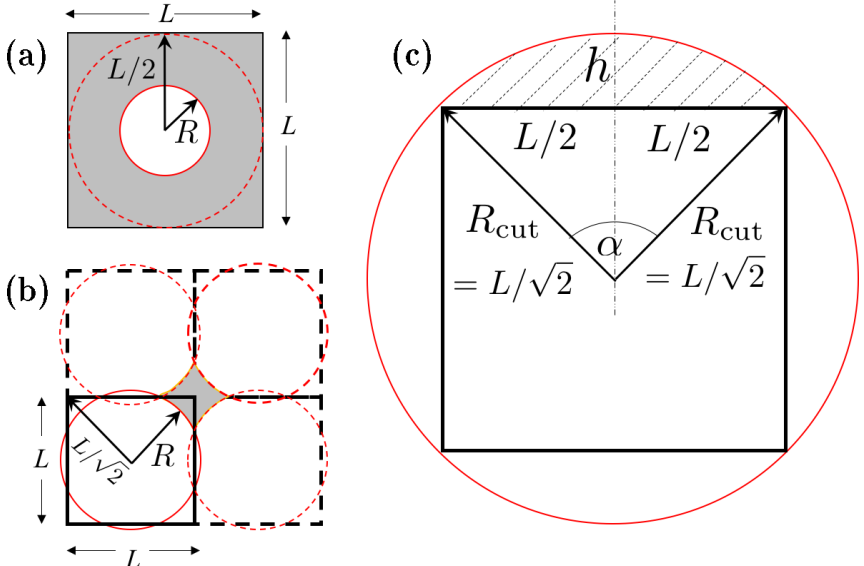

FIG. 10. Schematic illustration for defining the cutoff radius $R_{\text {cut }}$ for iteratively solving the modified Maxwell-Garnett equations. (a) Particle embedded in the matrix, (b) matrix embedded between the particles, (c) the limiting case defining the cutoff radius.

to that of a matrix embedded between the particles. In this case, the multishell Maxwell-Garnett model was found to be possible to adapt accordingly by reversing the particle fractions and segregation factors, computing them with respect to the particles unlike the case for Fig. 10(a). Finally, for $R_{\text {cut }}>$ $L / \sqrt{2}$, the matrix vanishes completely due to overlapping particles and the effective particle fraction becomes unity, a limiting case where the multishell Maxwell-Garnett approach does not apply. It is evident that if $R_{\text {cut }} \rightarrow \infty$ the effective diffusivity in the model will simply approach the diffusivity of the unstressed boundary, since the impact of a single precipitate on the diffusivity of an infinite sphere around it will be negligible.

\section{APPENDIX B: DETERMINING THE CRITICAL PRECIPITATE SIZE FOR COHERENCY LOSS}

A precipitate of radius $r$ embedded coherently in the matrix results in the elastic strain energy $E_{\text {str }}$ of the precipitate-matrix system. On the one hand, the coherency loss is accompanied by a decrease in the elastic strain energy. On the other hand, an interfacial dislocation is introduced at the precipitate-matrix interface accompanied by an increase in the interfacial energy $E_{\text {int }}$. The critical precipitate size $r_{\text {crit }}$ is determined from the energy conservation by equating the two $[48,49]$ :

$$
\underbrace{8 \pi r_{\mathrm{crit}}^{3} G \delta^{2} \frac{1+v}{3(1-v)}}_{E_{\mathrm{str}}}=\underbrace{4 \pi r_{\mathrm{crit}}^{2} \sigma_{\mathrm{dis}}}_{E_{\mathrm{int}}},
$$

where $G$ and $v$ are the shear modulus and Poisson's ratio of the matrix, respectively, and $\sigma_{\text {dis }}$ is the energy (per unit area) of the interfacial dislocation network defined as

$$
\begin{aligned}
\sigma_{\mathrm{dis}}= & \frac{G|\vec{b}(T)|}{2 \pi^{2}} \\
& \times[\underbrace{1+\beta-\sqrt{\left(1+\beta^{2}\right)}-\beta \ln \left\{2 \beta \sqrt{\left(1+\beta^{2}\right)}-2 \beta^{2}\right\}}_{X(T)}],
\end{aligned}
$$

where $\vec{b}$ is the Burgers vector of Al matrix $\left(|\vec{b}|=b_{110}=\frac{a_{\mathrm{m}} \sqrt{2}}{2}\right.$, $a_{\mathrm{m}}$ is the lattice parameter of $\left.\mathrm{Al}\right), \beta=\pi \delta^{\prime}(T) /(1-v)$, and $\delta^{\prime}(T)$ is the reduced (residual) coherency strain as a result of the introduction of interfacial misfit dislocations and is taken to be approximately equal to $\delta$. Solving (B1) yields the following expression of the temperature dependent critical size for coherency loss:

$$
r_{\text {crit }}(T)=\frac{|\vec{b}(T)|}{\delta^{2}(T)} \frac{3(1-v)}{4 \pi^{2}(1+v)} X(T) .
$$

The temperature dependence of $r_{\text {crit }}$ results from the temperature dependent lattice misfit and the Burgers vector where we have considered all the finite-temperature contributions including phonon-phonon anharmonicity. The typical value [95] of $v$ for $\mathrm{Al}$ is around 0.34 .

\section{APPENDIX C: SQUARE LATTICE MODEL TO DETERMINE THE RESIDUAL COHERENCY STRAIN}

The residual coherency strain $\delta^{\prime}$ due to the formation of interfacial dislocation at the matrix-precipitate interface is determined using the square lattice model $[74,75]$. Considering the ground state lattice parameters of $\mathrm{Al}(4.04 \AA)$ and $\mathrm{Al}_{3} \mathrm{Sc}$ (4.103 $\AA$ ), 65 units cells of $\mathrm{Al}$ and 64 unit cells of $\mathrm{Al}_{3} \mathrm{Sc}$ are needed to form a coherent interface between the two. Figure 11 schematically represents the square lattice consisting of an array of $65 \times 65$ unit cells with the dislocation core spread over seven atomic layers. This square lattice corresponds to a dislocation spacing of approximately $26.6 \mathrm{~nm}$ $(65 \times 4.103 \AA)$.

The coherent interfacial energy of $\mathrm{Al} / \mathrm{Al}_{3} \mathrm{Sc}$ interface comprises of chemical and strain contributions,

$$
\gamma_{\mathrm{coh}}=\gamma_{\mathrm{strain}}+\gamma_{\mathrm{chem}}
$$

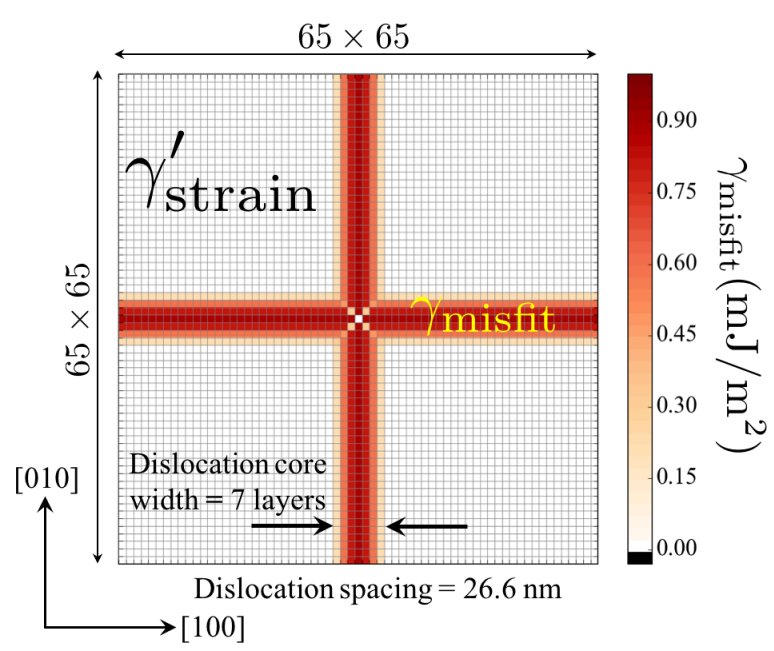

FIG. 11. A $65 \times 65$ square lattice model to determine the residual coherency strain due to the formation of misfit dislocation at the $\mathrm{Al} / \mathrm{Al}_{3} \mathrm{Sc}$ interface. The $\gamma_{\text {misfit }}$ values along the vertical and horizontal colored regions correspond to the $\gamma$-surface values along $\langle 100\rangle$ directions and the $\gamma_{\text {misfit }}$ values along the intersection points (forming $x$ in the center of the square lattice) of the dislocations correspond to the $\gamma$-surface values along $\langle 110\rangle$ directions. 
where the strain contribution arises due to the biaxial deformations of both the phases at the interface to adapt to a common in-plane lattice parameter in order to maintain the coherency. The introduction of a misfit dislocation at the interface reduces the coherency strain resulting in a semicoherent interface and the corresponding interfacial energy becomes

$$
\gamma_{\text {semi }}=\gamma_{\text {strain }}^{\prime}+\gamma_{\text {chem }}+\gamma_{\text {misfit }},
$$

where $\gamma_{\text {strain }}^{\prime}$ is the residual coherency-strain contribution and $\gamma_{\text {misfit }}$ the energy due to the presence of a misfit dislocation. The residual coherency strain $\left(\delta^{\prime}\right)$ is computed by dividing the number of white squares (representing the coherent regions) in Fig. 11 by the total number of unit cells in the square lattice $(65 \times 65$ in the present case $)$. Within the framework of square lattice model, the value of $\delta^{\prime}$ will of course vary with the dislocation core width and the dislocation spacing. For a dislocation core spread over seven atomic layers (Fig. 11), the residual coherency strain reduces to $80 \%, \delta^{\prime} \approx 0.8 \delta$.

The misfit energy $\gamma_{\text {misfit }}$ is computed from the $\gamma$ surface obtained from $a b$ initio calculations. For further technical details related to the $\gamma$ surface and the square lattice model, we refer to Refs. [74,75].
[1] R. Valiev, Y. Estrin, Z. Horita, T. G. Langdon, M. J. Zehetbauer, and Y. T. Zhu, JOM 58, 33 (2006).

[2] R. Valiev, R. K. Islamgaliev, and I. V. Alexandrov, Prog. Mater. Sci. 45, 103 (2000).

[3] M. J. Zehetbauer, R. Grössinger, H. Krenn, M. Krystian, R. Pippan, P. Rogl, T. Waitz, and R. Würschum, Adv. Eng. Mater. 12, 692 (2010).

[4] A. Mogucheva, D. Tagirova, and R. Kaibyshev, Mater. Sci. Forum 735, 353 (2013).

[5] C. Schwarze, A. Gupta, T. Hickel, and R. Darvishi Kamachali, Phys. Rev. B 95, 174101 (2017).

[6] J. Røyset and N. Ryum, Int. Mater. Reviews 50, 19 (2005).

[7] Yu. Buranova, V. Kulitskiy, M. Peterlechner, A. Mogucheva, R. Kaibyshev, S. V. Divinski, and G. Wilde, Acta Mater. 124, 210 (2017).

[8] G. M. Hood, R. J. Schultz, and J. Armstrong, Phil. Mag. A 47, 775 (1983).

[9] G. Erdélyi, D. L. Beke, F. J. Kedves, and I. Gödény, Phil. Mag. B 38, 445 (1978).

[10] N. L. Peterson and S. J. Rothman, Phys. Rev. B 1, 3264 (1970).

[11] M. S. Anand and R. P. Agarwala, Phil. Mag. 26, 297 (1972).

[12] G. Rummel, T. Zumkley, M. Eggersmann, K. Freitag, and H. Mehrer, Z. Metallkd. 86, 122 (1995).

[13] A. D. Le Claire, J. Nucl. Mater. 69-70, 70 (1978).

[14] S. V. Divinski, G. Reglitz, H. Rösner, Y. Estrin, and G. Wilde, Acta Mater. 59, 1974 (2011).

[15] J. Fiebig, S. V. Divinski, H. Rösner, Y. Estrin, and G. Wilde, J. Appl. Phys. 110, 083514 (2011).

[16] Chr. Herzig and S. V. Divinski, Materials Transaction (JIM) 44, 14 (2003).

[17] S. V. Divinski, Diffusion Foundations 5, 57 (2015).

[18] X. Sauvage, G. Wilde, S. V. Divinski, Z. Horita, and R. Z. Valiev, Mat. Sci. Eng. A 540, 1 (2012).

[19] M. M. Abramova, N. A. Enikeev, X. Sauvage, A. Etienne, B. Radiguet, E. Ubyivovk, and R. Z. Valiev, Rev. Adv. Mater. Sci. 43, 83 (2015).

[20] D. Setman, M. B. Kerber, E. Schafler, and M. J. Zehetbauer, Metall. Mater. Trans. A 41, 810 (2009).

[21] Y. Estrin and Y. Vinogradov, Acta Mater. 61, 782 (2013).

[22] A. A. Nazarov, A. E. Romanov, and R. Z. Valiev, Acta Metall. Mater. 41, 1033 (1993).

[23] M. W. Grabski and R. Korski, Philos. Mag. 22, 707 (1970).

[24] R. A. Varin, J. W. Wyrzykowski, W. Łojkowski, and M. W. Grabski, phys. stat. sol. (a) 45, 565 (1978).

[25] X.-Y. Liu and J. Adams, Acta Mater. 46, 3467 (1998).
[26] X. Sauvage, E. Bobruk, M. Y. Murashkin, Y. Nasedkina, N. Enikeev, and R. Valiev, Acta Mater. 98, 355 (2015).

[27] A. Mogucheva, E. Babich, B. Ovsyannikov, and R. Kaibyshev, Mat. Sci. Eng. A 560, 178 (2013).

[28] F. Humphreys and M. Hatherly, J. Microscopy 213, 247 (2004).

[29] S. Malopheyev, S. Mironov, V. Kulitskiy, and R. Kaibyshev, Mat. Sci. Eng. A 624, 132 (2015).

[30] R. Kaibyshev, K. Shipilova, F. Musin, and Y. Motohashi, Mat. Sci. Eng. A 396, 341 (2005).

[31] V. Kulitskiy, S. Malopheyev, Yu. Buranova, S. V. Divinski, G. Wilde, and R. Kaibyshev, Mater. Sci. Forum 838, 379 (2016).

[32] L. G. Harrison, Trans. Faraday Soc. 57, 1191 (1961).

[33] A. Paul, T. Laurila, V. Vuorinen, and S. V. Divinski, Thermodynamics, Diffusion and the Kirkendall Effect in Solids (Springer Int. Publ., Switzerland, 2014).

[34] H. Mehrer, Diffusion in Solids: Fundamentals, Methods, Materials, Diffusion-Controlled Processes (Springer, Berlin, 2007).

[35] D. L. Beke, I. Gödény, G. Erdélyi, and F. J. Kedves, Phil. Mag. A 56, 659 (1987).

[36] G. Kresse and J. Hafner, Phys. Rev. B 47, 558 (1993).

[37] G. Kresse and J. Furthmüller, Phys. Rev. B 54, 11169 (1996).

[38] J. P. Perdew, K. Burke, and M. Ernzerhof, Phys. Rev. Lett. 77, 3865 (1996).

[39] H. J. Monkhorst and J. D. Pack, Phys. Rev. B 13, 5188 (1976).

[40] M. Methfessel and A. T. Paxton, Phys. Rev. B 40, 3616 (1989).

[41] G. Henkelman, B. P. Uberuaga, and H. Jónsson, J. Chem. Phys. 113, 9901 (2000).

[42] G. Henkelman and H. Jónsson, J. Chem. Phys. 113, 9978 (2000).

[43] http://theory.cm.utexas.edu/vtsttools/.

[44] D. Prokoshkina, V. Esin, G. Wilde, and S. V. Divinski, Acta Mater. 61, 5188 (2013).

[45] S. V. Divinski and B. S. Bokstein, Defect Diffusion Forum 309310, 1 (2011).

[46] D. Liu, J. Fiebig, M. Peterlechner, S. Trubel, M. Wegner, Y. Du, Zh. Jin, G. Wilde, and S. V. Divinski, Intermetallics 61, 30 (2015).

[47] H. Kang, X. Li, Y. Su, D. Liu, J. Guo, and H. Fu, Intermetallics 23, 32 (2012).

[48] S. Iwamura and Y. Miura, Acta Mater. 52, 591 (2004).

[49] S. Saha, T. Z. Todorova, and J. W. Zwanziger, Acta Mater. 89, 109 (2015).

[50] C. Watanabe, T. Kondo, and R. Monzen, Metall. Mater. Trans. A 35A, 3003 (2004).

[51] Y. W. Riddle and T. H. Sanders, Jr., Mater. Sci. Forum A 331337, 939 (2000). 
[52] M. J. Jones and F. J. Humphreys, Acta Mater. 51, 2149 (2003).

[53] A. L. Berezina, V. A. Volkov, B. P. Domashnikov, S. V. Ivanov, and K. V. Chuistov, Phys. Met. 10, 296 (1990).

[54] Y. W. Riddle and T. H. Sanders, Jr., Metall. Mater. Trans. A 35, 341 (2004).

[55] E. A. Marquis and D. N. Seidman, Acta Mater. 49, 1909 (2001).

[56] J. L. Murray, Bull. Alloy Ph. Diag. 3, 60 (1982).

[57] Y. Harada and D. C. Dunand, Mat. Sci. Eng. A 329, 686 (2002).

[58] J. R. Kalnin, E. A. Kotomin, and J. Maier, J. Phys. Chem. Solids 63, 449 (2002).

[59] I. V. Belova and G. E. Murch, J. Phys. Chem. Solids 64, 873 (2003).

[60] J. D. Eshelby, Proc. Royal Soc. London A 241, 376 (1957).

[61] A. Gupta, B. T. Kavakbasi, B. Dutta, B. Grabowski, M. Peterlechner, T. Hickel, S. V. Divinski, G. Wilde, and J. Neugebauer, Phys. Rev. B 95, 094307 (2017).

[62] A. Mogucheva, D. Yuzbekova, T. Lebedkina, M. Lebyodkin, and R. Kaibyshev, Mater. Sci. Forum 879, 1317 (2017).

[63] R. Swiatek, M. Zehetbauer, and B. Mikulowski, Mat. Sci. Eng. A 234, 441 (1997).

[64] A. D. LeClaire and A. B. Lidiard, Philos. Mag. 1, 518 (1956).

[65] W. Triftshäuser, Phys. Rev. B 12, 4634 (1975).

[66] M. J. Fluss, L. C. Smedskjaer, M. K. Chason, D. G. Legnini, and R. W. Siegel, Phys. Rev. B 17, 3444 (1978).

[67] T. M. Hall, A. N. Goland, and C. L. Snead, Jr., Phys. Rev. B 10, 3062 (1974).

[68] B. T. A. McKee, A. G. D. Jost, and I. K. MacKenzie, Can. J. Phys. 50, 415 (1972).

[69] N. Sandberg and R. Holmestad, Phys. Rev. B 73, 014108 (2006).

[70] D. Simonovic and M. H. F. Sluiter, Phys. Rev. B 79, 054304 (2009).

[71] C. Wolverton, Acta Mater. 55, 5867 (2007).

[72] As per the definitions in Refs. [70], the positive values of solutevacancy binding energy represent attractive interaction. For a direct comparison with our definition of binding energy, we have indicated the values of Refs. [70] with opposite signs.

[73] E. A. Marquis, D. N. Seidman, M. Asta, C. Woodward, and V. Ozolinš, Phys. Rev. Lett. 91, 036101 (2003).
[74] J. M. Albina, M. Mrovec, B. Meyer, and C. Elsässer, Proc. of 3rd Int. Conf. Multiscale Materials Modeling (Fraunhofer IRB Verlag, Stuttgart, 2006), Vol. 18-22, p. 819.

[75] S. Sampath and R. Janisch, J. Phys.: Condens. Matter 25, 355005 (2013).

[76] T. Surholt, Y. M. Mishin, and C. Herzig, Phys. Rev. B 50, 3577 (1994).

[77] S. V. Divinski, G. Reglitz, and G. Wilde, Acta Mater. 58, 386 (2010).

[78] Z. Tokei, Z. Erdelyi, C. Girardeaux, and A. Rolland, Phil. Mag. A 80, 1075 (2000).

[79] X. Sauvage, N. Enikeev, R. Valiev, Y. Nasedkina, and M. Murashkin, Acta Mater. 72, 125 (2014).

[80] S. J. Dillon, M. Tang, W. C. Carter, and M. P. Harmer, Acta Mater. 55, 6208 (2007).

[81] H. Edelhoff, S. I. Prokofjev, and S. V. Divinski, Scripta Mater. 64, 374 (2011)

[82] S. V. Divinski, H. Edelhoff, and S. Prokofjev, Phys. Rev. B 85, 144104 (2012).

[83] T. Frolov, S. V. Divinski, M. Asta, and Y. Mishin, Phys. Rev. Lett. 110, 255502 (2013).

[84] T. Frolov, M. Asta, and Y. Mishin, Phys. Rev. B 92, 020103(R) (2015).

[85] A. A. Nazarov, Interface Sci. 8, 315 (2000).

[86] A. Häßner, Kristall und Technik 9, 1371 (1974).

[87] M. B. Kerber, M. J. Zehetbauer, E. Schafler, F. C. Spieckermann, S. Bernstorff, and T. Ungar, JOM 63, 61 (2011).

[88] K. R. Cardoso, M. A. Munoz-Morris, K. Valdes Leon, and D. G. Morris, Mater. Sci. Eng. A 587, 387 (2013).

[89] S. V. Divinski, J. Geise, E. Rabkin, and Chr. Herzig, Z. Metallkunde 95, 945 (2004).

[90] J. Sommer and Chr. Herzig, J. Appl. Phys. 72, 2758 (1992).

[91] S. V. Divinski, F. Hisker, Y.-S. Kang, J.-S. Lee, and Chr. Herzig, Z. Metallkde. 93, 256 (2002).

[92] S. V. Divinski, F. Hisker, Y.-S. Kang, J.-S. Lee, and Chr. Herzig, Z. Metallkde. 93, 265 (2002).

[93] L. Klinger and E. Rabkin, Acta Mater. 47, 725 (1999).

[94] I. A. Szabo, D. L. Beke, and F. J. Kedves, Phil. Mag. 62, 227 (1990).

[95] C. Comte and J. von Stebut, Surf. and Coat. Tech. 154, 42 (2002). 